HD-THEP-04-49

IFUM-792-FT

hep-ph/0501192

\title{
The $C$-Odd Four-Gluon State in the Color Glass Condensate
}

\author{
Stefan Braunewell ${ }^{a, b, 1}$, Carlo Ewerz ${ }^{a, c, 2}$ \\ ${ }^{a}$ Institut für Theoretische Physik, Universität Heidelberg \\ Philosophenweg 16, D-69120 Heidelberg, Germany \\ ${ }^{b}$ Institut für Theoretische Physik, Universität Bremen \\ Otto-Hahn-Allee 1, D-28359 Bremen, Germany \\ ${ }^{c}$ Dipartimento di Fisica, Università di Milano and INFN, Sezione di Milano \\ Via Celoria 16, I-20133 Milano, Italy
}

\begin{abstract}
The study of the perturbative Odderon at high gluon densities in the color glass condensate requires to take into account states with more than three gluons. We formulate evolution equations for these states in which the number of gluons is not fixed during the evolution. We further determine the coupling of these Odderon states to the $\gamma \rightarrow \eta_{c}$ impact factor for arbitrary numbers of gluons. We find an exact solution of the evolution equation for the four-gluon Odderon state in terms of the three-gluon Odderon state. It is shown that a similar solution exists also for a different class of Odderon solutions which does not couple to the $\gamma \rightarrow \eta_{c}$ impact factor. We discuss the implications of our result from the perspective of constructing an effective field theory of reggeized gluons for the color glass condensate.
\end{abstract}

${ }^{1}$ email: braunewell@itp.uni-bremen.de

2 email: Carlo.Ewerz@mi.infn.it 


\section{Introduction}

In high energy collisions the partons inside hadrons form a very dense system which is often called the color glass condensate. At sufficiently high density the partons overlap such that recombination effects become important and tend to slow down the growth of the density with increasing energy. It is expected that eventually a saturation regime is reached. The theoretical challenge is to understand at which energies and how this takes place in detail. In order to answer this question one formulates evolution equations describing the behavior of the system with increasing energy. The color glass condensate has been studied in a number of different perturbative approaches which are applicable if the scattering process involves a hard scale. Prominent examples are the approach initiated by McLerran and Venugopalan [1, 2] (for a review see [3]), the operator expansion of Wilson lines due to Balitsky [4, or the color dipole picture of high energy scattering developed by Mueller [5, 6]. Most of these approaches lead to similar results in suitable approximations. They all reproduce for example the BalitskyKovchegov (BK) evolution equation which resums certain classes of multi-Pomeron exchanges 4, 7, 8, 9, and which has become a key tool in investigating the color glass condensate and in particular potential saturation effects at high energies.

The approach which we will use in the present paper is the generalized leading logarithmic approximation (GLLA) [10]-13]. It is an extension of the classical approach to high energy scattering in QCD based on the resummation of large logarithms that in leading logarithmic order (LLA) gives rise to the BFKL Pomeron [14, 15]. The BFKL Pomeron can be viewed as the exchange of two interacting reggeized gluons. In the GLLA one goes beyond that approximation by taking into account also exchanges of more than two gluons, and one collects all perturbative contributions that contain the maximally possible number of logarithms for a given number of gluons. There are two versions of the GLLA: in the first version the number of gluons exchanged in the $t$-channel of the scattering process remains constant, whereas in the second version the number of gluons is allowed to change during the $t$-channel evolution [12]. The latter is often called extended GLLA, or EGLLA. It is the fluctuation of the number of gluons in the $t$-channel which in the resummation approach reflects the parton recombination effects characterizing the color glass condensate. Different aspects of the EGLLA have been studied in [16]-29]. There it has been found that in the Pomeron channel, i. e. for the exchange of even $C$-parity, the EGLLA gives rise to a picture of an effective field theory in which states consisting of even numbers of gluons are coupled to each other via effective vertices. So far the vertices from two to four [18] and from two to six gluons 24] have been calculated explicitly. From the two-to-four gluon vertex one obtains the perturbative triple Pomeron vertex [21], and in a similar way the one-to-three Pomeron vertex is obtained from the two-to-six gluon vertex [28]. Recently it has been shown that the triple Pomeron vertex obtained in this way gives rise to the BK equation when the so-called Möbius representation is used for the Pomerons 30. As discussed in that reference the EGLLA not only reproduces the BK equation but also makes it possible to compute subleading corrections to the BK equation which appear difficult to access in other approaches to the color glass condensate.

An advantage of the EGLLA is that the remarkable property of conformal invariance in two-dimensional impact parameter space [31, 20, 27] and the phenomenon of gluon reggeization [32] become particularly transparent in this approach. Especially the 
reggeization of the gluon will turn out to be crucial in our present investigation. The gluons exchanged in high energy scattering are reggeized, that is they are composite objects consisting themselves of reggeized gluons in a selfconsistent way. A manifestation of this phenomenon is that whenever two $t$-channel gluons are at the same point in impact parameter space they behave like a single gluon. When this occurs explicitly in a scattering amplitude we can hence see how the composite gluon is formed out of the two gluons. The same picture can be extended to more than two gluons, and one can systematically resolve higher Fock states of the reggeized gluon in the framework of the EGLLA, see [26].

So far the color glass condensate has been studied almost exclusively in the Pomeron channel, that is for the exchange of vacuum quantum numbers (in particular for positive $C$-parity) which is relevant to total cross sections. In the present paper we make a first step of a systematic study of the Odderon in the EGLLA, that is for the channel in which negative charge parity quantum number is exchanged. In lowest order in the GLLA the Odderon is an exchange of three interacting gluons in the $t$-channel in a symmetric color state, and is described by the Bartels-Kwieciński-Praszałowicz (BKP) equation [11, 13. Two types of explicit solutions to the BKP equation have been found in [33] and 34, respectively. The latter solution has also been found in the dipole picture of high energy scattering in [35] where also some potential effects of the color glass condensate on the Odderon are discussed. For a review of the theory and phenomenology of the Odderon see 36].

Our motivation for studying also the Odderon in the EGLLA is twofold. Firstly, states with more than three gluons in the $t$-channel might be important phenomenologically. One could for example expect that Pomeron loops can affect the Odderon intercept. Recall that the intercept of the perturbative Odderon is close to one (and exactly one for the solution of 34]). Hence potential corrections to the intercept due to Pomerons would be particularly significant. Another interesting effect is the interplay of Pomeron and Odderon exchanges in high energy scattering. Some possible effects of that interplay have recently been discussed in [37. A detailed study certainly requires a concise knowledge of the splitting of an Odderon into an Odderon and a Pomeron, as it can be obtained in the EGLLA. The calculation of that vertex is among the goals of the investigation that we initiate in the present paper. We expect that this vertex will also make it possible to obtain the large- $N_{c}$ limit of the EGLLA and thus give rise to an equation for the Odderon channel which is the analog of the BK equation for the Pomeron channel. This equation should then be of a similar form as the one suggested in the context of saturation in the dipole picture for the Odderon in 35.

An equally important motivation for studying the Odderon in the EGLLA is to gain a better understanding of the effective theory of the color glass condensate and of its properties. Important aspects in this respect would for instance be an investigation of the crossing properties of the vertices in the effective field theory and their interpretation in view of conformal field theories. In addition, studying Odderon states will hopefully also make it possible to compute the gluon vertices of the effective field theory - which so far are known only for color singlet channels - in arbitrary color states.

In a first step we consider in the present paper the case of up to four gluons. We give explicitly the coupled evolution equations for the three- and four-gluon states. As an initial condition for the evolution we choose the coupling of the Odderon to the 
$\gamma \rightarrow \eta_{c}$ impact factor. This impact factor can be computed in perturbation theory due to the large mass of the charm quark. It is also of phenomenological relevance, see for example [38- [42]. We will show that the impact factor exhibits reggeization. That fact will make it possible to find an explicit solution of the evolution equation for the fourgluon state when it is coupled to the $\gamma \rightarrow \eta_{c}$ impact factor. Due to that impact factor, the Odderon solution found in [34] is projected out. We will show that an analogous result can be obtained for the solution of [33] by considering the case of a baryonic impact factor. As we will discuss in the conclusions, our result lays the foundations for a future investigation of the five-gluon Odderon state in which we expect new elements of the effective field theory to occur.

We should mention that Odderon states with more than three gluons have already been discussed in the GLLA, but only with the number of gluons being kept fixed during the evolution. There a remarkable simplification takes place in the large- $N_{c}$ limit in which these $n$-gluon states are equivalent to an integrable XXX Heisenberg model [43- 45]. It should be pointed out, however, that for $n>3$ the $n$-gluon states obtained in the large- $N_{c}$ limit are not the leading ones at high energies. Moreover, they cannot be coupled directly to the phenomenologically interesting impact factors, as will become clear also in our discussion below. We emphasize that in the present paper we do not fix the number of gluons in the $t$-channel evolution, and also do not take the large- $N_{c}$ limit.

The paper is organized as follows. In section 2 we formulate the evolution equations for the $n$-gluon Odderon states in the EGLLA. We then compute the $\gamma \eta_{c^{-}}$Odderon impact factor with an arbitrary number $n$ of gluons $(n \geq 3)$ and give the result explicitly for the cases of four and five gluons. The result is expressed in terms of the impact factor with only three gluons. Based on that observation we are then able to find an analytic solution of the four-gluon Odderon state in the EGLLA and discuss its properties in section [4. In section [5] we generalize our result to other impact factors and hence to all known types of Odderon solutions. Our results are summarized in section [6. In the course of our calculation we also find some useful results for the $C$-even channel which we present in an appendix.

\section{Coupled evolution equations for the Odderon in EGLLA}

We start with the well-known BKP equation for a system of three interacting gluons exchanged in the $t$-channel of a scattering process [11, 13]. In the case of the Odderon the three gluons are in a state that is odd under $C$-parity. We consider the Odderon amplitude coupled to the $\gamma \rightarrow \eta_{c}$ impact factor, which hence constitutes the initial condition for the evolution of the Odderon state in rapidity. We define $F_{3}$ as the amputated three-gluon Odderon amplitude with discontinuities taken in the squared energies obtained from the four-momenta of the photon and the first gluon, and of the photon and the first and second gluon, respectively. At high energies, the dynamics effectively reduces to the transverse plane of the reaction, see section 3.1 below. The amplitude $F_{3}$ accordingly depends on the transverse momenta of the three gluons, and it obviously carries color labels for the gluons. In addition, $F_{3}$ depends on the momenta of the photon and the $\eta_{c}$-meson, but we will suppress this dependence in our notation. It is convenient to change from the squared center-of-mass energy $s$ to complex angular 
momentum $\omega$ via a Sommerfeld-Watson transformation.

The BKP equation in transverse momentum space then reads

$$
\left(\omega-\sum_{i=1}^{3} \beta\left(\mathbf{k}_{i}\right)\right) F_{3}^{a_{1} a_{2} a_{3}}=F_{(3 ; 0)}^{a_{1} a_{2} a_{3}}+\sum K_{2 \rightarrow 2}^{\{b\} \rightarrow\{a\}} \otimes F_{3}^{b_{1} b_{2} b_{3}},
$$

with the gluon trajectory function $\beta$ describing virtual corrections to the $t$-channel gluons,

$$
\beta\left(\mathbf{k}^{2}\right)=-\frac{N_{c}}{2} g^{2} \int \frac{d^{2} \mathbf{l}}{(2 \pi)^{3}} \frac{\mathbf{k}^{2}}{\mathbf{l}^{2}(\mathbf{l}-\mathbf{k})^{2}},
$$

where the bold-face characters denote two-dimensional transverse momenta. The BKP equation can be viewed as a Schrödinger type equation with $\omega$ being the energy-like variable. The conjugate time-like variable is rapidity $Y=\log \left(s / s_{0}\right)$, where $s_{0}$ is a fixed hadronic energy scale. The kernel $K_{2 \rightarrow 2}^{\{b\} \rightarrow\{a\}}$ describes the pairwise interaction of the gluons. It is up to a color factor identical to the part of the integral kernel of the BFKL equation which describes real gluon production. We will give an explicit representation of the kernel as well as an explanation of the convolution symbol further below. In the case of three gluons one can separate the color and the momentum part,

$$
F_{3}^{a_{1} a_{2} a_{3}}\left(\mathbf{k}_{1}, \mathbf{k}_{2}, \mathbf{k}_{3}\right)=d_{a_{1} a_{2} a_{3}} F_{3}\left(\mathbf{k}_{1}, \mathbf{k}_{2}, \mathbf{k}_{3}\right),
$$

with the symmetric structure constant $d_{a_{1} a_{2} a_{3}}$ of the $\mathrm{SU}(3)$ color group. We will sometimes suppress the momentum arguments of the function $F_{3}$.

The inhomogeneous term $F_{(3 ; 0)}$ in the BKP equation (11) is given by the impact factor of the transition $\gamma \rightarrow \eta_{c}$. We refer the reader to 38] for the explicit formula for the impact factor with three gluons. Its explicit form will not be needed for our discussion. We only note here that the impact factor $F_{(3 ; 0)}$ is symmetric in the three gluon momenta and in their color labels, and that it vanishes due to gauge invariance when one of the gluon momenta vanishes. It should be pointed out that our particular choice of the $\gamma \rightarrow \eta_{c}$ impact factor singles out a particular solution to the BKP equation, namely the Bartels-Lipatov-Vacca (BLV) solution 34. That solution is a superposition of states depending only on two transverse coordinates. In contrast to that situation, the other known class of solutions of the BKP equation requires the three gluons to be at different positions in transverse space and vanishes when two of the three gluon positions coincide. (The Janik-Wosiek solution found in 33. belongs to this class.) In the $\gamma \rightarrow \eta_{c}$ impact factor in leading order the photon splits into a quark-antiquark pair to which the three gluons couple, and which then recombines into an $\eta_{c}$ meson. The intermediate state with only two quarks provides only two points in transverse space to which the gluons couple and hence singles out the BLV solution. We will first concentrate on that solution and will come back to the other class of solutions in section 5

The symmetry of the impact factor mentioned above immediately allows us to find a crucial property of the full three-gluon amplitude without even using the particular form of the BLV solution, namely the separate invariance of the amplitude under exchange of the momenta and of the color indices. The latter observation is trivial because of the color tensor $d_{a_{1} a_{2} a_{3}}$ which the Odderon keeps throughout its evolution. The invariance under momentum-exchange can be inferred from the BKP equation (11) as the quark 
loop has this property and the application of the integral kernel respects the symmetry because the sum over all pairwise interactions is performed. Formally constructing the solution by iterating the integral equation then leads to the conclusion that this property transfers to the full three-gluon Odderon amplitude. Of course, this symmetry property is also reflected in the explicit formula of the BLV solution, see [34. Later we will need only a slightly weaker property of the three-gluon Odderon state, namely its symmetry under simultaneous exchange of momentum and color labels which is a trivial consequence of the separate symmetries in color and momentum space. In a similar way one can start from the fact that the impact factor vanishes for vanishing gluon momenta and derive that also the full amplitude $F_{3}$ vanishes if one of the three gluon momenta vanishes.

We now want to write the BKP equation in a diagrammatic form in order to make the generalization to higher gluon numbers more transparent,

$$
\left(\omega-\sum_{i=1}^{3} \beta\left(\mathbf{k}_{i}\right)\right) \stackrel{-F_{3}}{F_{3}}+\sum \begin{gathered}
F_{3} \\
\prod_{1}
\end{gathered} .
$$

In this notation, the diagrams representing the impact factor and the full Odderon amplitude include the color tensors and the contributions from the external particles, but are amputated, i.e. the 'outgoing' gluon propagators are cut off. The kernel that acts on the amplitude also includes color tensors which we give explicitly below. The sum in the last term extends over all pairwise interactions of the gluons.

It is now straightforward to apply the EGLLA to the $C$-odd channel following the same procedure which has been developed in [11, 12] for the $C$-even (Pomeron) channel. The lowest possible contribution in the $C$-odd channel is the Odderon with three gluons satisfying the BKP equation, see above. In the EGLLA, we have to take into account in addition all exchanges with more than three gluons, and have to allow for numberchanging transitions during the evolution. This is most conveniently implemented in the form of integral equations that generalize the BKP equation. (For a detailed discussion of the corresponding integral equations in the $C$-even channel see 24.) In these integral equations the number-changing transitions are due to integral kernels $K_{2 \rightarrow m}$ which have been derived in 12. Note that as a result of the approximation scheme of the EGLLA in these kernels only two gluons interact with each other to produce more $t$-channel gluons. This is similar to the BKP equation (11), where in each contribution to the last term only two gluons undergo an interaction via the BFKL kernel. In the amplitude for an $n$-gluon state one then has to take into account all possible contributions in which a state with $l$ gluons $(l<n)$ undergoes a transition to an $n$-gluon state via a kernel $K_{2 \rightarrow m}$ with $m=n-l+2$, and we have to include all possible $l$ starting from the lowest possible amplitude that has $l=3$. For us it is important that the derivation [12] of the kernels $K_{2 \rightarrow m}$ for the interaction of two gluons does not require any assumptions about the other gluons, in particular it does not require a specific $C$-parity of the whole $n$-gluon state. We can therefore use exactly the same kernels to formulate the integral equations for $n$-gluon amplitudes also in the $C$-odd channel.

In this way one obtains the following integral equation for the $C$-odd four-gluon 
amplitude $F_{4}$ in the EGLLA,

$$
\begin{aligned}
\left(\omega-\sum_{i=1}^{4} \beta\left(\mathbf{k}_{i}\right)\right) F_{4}^{a_{1} a_{2} a_{3} a_{4}}= & F_{(4 ; 0)}^{a_{1} a_{2} a_{3} a_{4}}+\sum K_{2 \rightarrow 3}^{\{b\} \rightarrow\{a\}} \otimes F_{3}^{b_{1} b_{2} b_{3}} \\
& +\sum K_{2 \rightarrow 2}^{\{b\} \rightarrow\{a\}} \otimes F_{4}^{b_{1} b_{2} b_{3} b_{4}}
\end{aligned}
$$

which in diagrammatic notation reads:

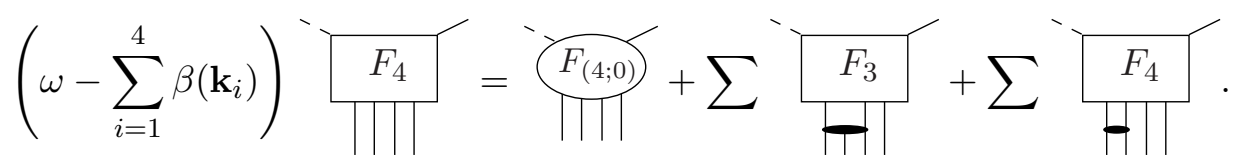

One can easily construct also the equations for the higher $n$-gluon amplitudes $F_{n}$, again in complete analogy to the case of even $C$-parity. For $n=5$, for example, the equation reads

$$
\begin{aligned}
\left(\omega-\sum_{i=1}^{5} \beta\left(\mathbf{k}_{i}\right)\right) F_{5}^{a_{1} a_{2} a_{3} a_{4} a_{5}}= & F_{(5 ; 0)}^{a_{1} a_{2} a_{3} a_{4} a_{5}}+\sum K_{2 \rightarrow 4}^{\{b\} \rightarrow\{a\}} \otimes F_{3}^{b_{1} b_{2} b_{3}} \\
& +\sum K_{2 \rightarrow 3}^{\{b\} \rightarrow\{a\}} \otimes F_{4}^{b_{1} b_{2} b_{3} b_{4}} \\
& +\sum K_{2 \rightarrow 2}^{\{b\} \rightarrow\{a\}} \otimes F_{5}^{b_{1} b_{2} b_{3} b_{4} b_{5}} .
\end{aligned}
$$

In this way one obtains the full (infinite) hierarchy of coupled integral equations of the EGLLA for the $C$-odd channel.

To complete the account of the integral equations for the $C$-odd channel in EGLLA, we have to give the exact expression for the interaction kernels. As the $2 \rightarrow m$ kernels can be written in a general form, we can do this in one step for both kernels that appear up to the four-gluon equation. Let the two gluons that enter the kernel from above carry the transverse momenta $\mathbf{l}_{1}$ and $\mathbf{l}_{2}$ and the color labels $b_{1}$ and $b_{2}$, and let the $m$ outgoing gluons carry the momenta $\mathbf{k}_{1}, \ldots, \mathbf{k}_{m}$ and the color labels $a_{1}, \ldots, a_{m}$. The integral kernels for the transition of two to $m$ gluons then read [12] (the generalization to arbitrary indices being trivial)

$$
\begin{aligned}
K_{2 \rightarrow m}^{\{b\} \rightarrow\{a\}}\left(\mathbf{l}_{1}, \mathbf{l}_{2} ; \mathbf{k}_{1}, \ldots, \mathbf{k}_{m}\right)= & g^{m} f_{b_{1} a_{1} k_{1}} f_{k_{1} a_{2} k_{2}} \ldots f_{k_{m-1} a_{m} b_{2}} \\
& {\left[\left(\mathbf{k}_{1}+\cdots+\mathbf{k}_{m}\right)^{2}-\frac{\mathbf{l}_{2}^{2}\left(\mathbf{k}_{1}+\cdots+\mathbf{k}_{m-1}\right)^{2}}{\left(\mathbf{k}_{m}-\mathbf{l}_{2}\right)^{2}}\right.} \\
& \left.-\frac{\mathbf{l}_{1}^{2}\left(\mathbf{k}_{2}+\cdots+\mathbf{k}_{m}\right)^{2}}{\left(\mathbf{k}_{1}-\mathbf{l}_{1}\right)^{2}}+\frac{\mathbf{l}_{1}^{2} \mathbf{l}_{2}^{2}\left(\mathbf{k}_{2}+\cdots+\mathbf{k}_{m-1}\right)^{2}}{\left(\mathbf{k}_{1}-\mathbf{l}_{1}\right)^{2}\left(\mathbf{k}_{m}-\mathbf{l}_{2}\right)^{2}}\right] .
\end{aligned}
$$

For the $2 \rightarrow 2$ kernel $(m=2)$ the last term in the brackets is defined to be zero so that one gets the BFKL kernel without the virtual corrections. Finally, the convolution symbol $\otimes$ implies a factor $\left[(2 \pi)^{3} \mathbf{l}_{1}^{2} \mathbf{l}_{2}^{2}\right]^{-1}$ followed by an integration $\int d^{2} \mathbf{l}_{1}$ over the loop momentum. Clearly, there is a color factor $\delta^{a_{i} b_{j}}$ for every gluon that does not participate in the kernel interaction.

As in the case of $C$-even exchanges, the integral equations of the EGLLA as described above apply to cut amplitudes. The four-gluon amplitude $F_{4}$, for example, is a result of taking three discontinuities with respect to the energy variables obtained 
from the photon and the first $r$ gluons, $r=1,2,3$. It should be noted that, as a result of these discontinuities, the amplitude $F_{4}$ is not fully symmetric under the exchange of two gluons. The full Bose symmetry of the $n$-gluon states is only restored in the full amplitude reconstructed via dispersion relations. Instead, the cut amplitudes for which the integral equations are formulated obey a particular set of Ward-type identities found in [26] for the $C$-even channel. As we will discuss below, our result for $F_{4}$ satisfies these identities.

Finally, a remark is in order concerning the calculation of scattering amplitudes from the amplitudes $F_{n}$ of our integral equations. In order to calculate the amplitude for the quasidiffractive process $\gamma \gamma \rightarrow \eta_{c} \eta_{c}$ for three $t$-channel gluons, one would have to fold the solution of the BKP equation (11) simply with the lower impact factor after attaching simple gluon propagators (without interaction) to it as is illustrated in figure 2 below (see also [42]). However, for the higher gluon number Odderon equations such a simple reconstruction of the full scattering amplitude must fail, because the integral equation also includes the transition from three to four $t$-channel gluons but not the other way around, which would lead to an asymmetric treatment of the upper and lower impact factor. Simply taking the square of the result is also not possible because it would double count some of the contributions. In fact, the reconstruction of the physical amplitude for the process $\gamma \gamma \rightarrow \eta_{c} \eta_{c}$ from the solution $F_{n}$ of the integral equations for general $n$ is a nontrivial task and requires a very careful use of dispersion relations in order to undo the cuts applied to the amplitude. A detailed discussion of this problem is beyond the scope of the present paper. When considering other scattering processes like for example photon-proton scattering, however, the coupling of the four-gluon system directly to the scattering partner is easier and does not lead to the problems mentioned above.

\section{The $\gamma \boldsymbol{\eta}_{c^{-}}$Odderon impact factor}

We now turn to the calculation of the $\gamma \rightarrow \eta_{c}$ impact factor with an arbitrary number $n>3$ of gluons coupled to it in GLLA. Due to the quantum numbers of the photon and of the $\eta_{c}$ meson the $n$ gluons are always in a $C$-odd state.

It will be useful to recall first in a more general setting the factorization of scattering processes at high energies which gives rise to impact factors. After discussing the $\gamma \rightarrow \eta_{c}$ impact factor with three gluons we will then consider more gluons. For that the crucial point is to study the step from $n$ to $n+1$ gluons, that is the effect of attaching one additional gluon to the impact factor. We show that the corresponding diagrams with $n+1$ gluons can be expressed in terms of diagrams with $n$ gluons in a particular way. That step has been considered more or less explicitly in many studies before (see for example [19, 24]), but we find it useful to discuss it here in more detail in a notation suitable for our purposes. Using that result and taking into account the color algebra we are then able to find a general formula for our impact factor with $n$ gluons in terms of the three-gluon impact factor.

\subsection{High energy factorization and impact factors}

Let us consider the scattering amplitude for a perturbatively calculable process at high energies in the GLLA. To be specific, we choose the quasidiffractive process $\gamma \gamma \rightarrow \eta_{c} \eta_{c}$, 
but the discussion also holds for other processes. In the following derivation of the impact factor representation for the scattering amplitude we follow [46] (where the analysis was conducted for the QED case, i.e. photon exchange) and [47] (where the two-gluon QCD case is treated).

At high energies the scattering process is dominated by gluon exchange, the lowest order contribution being three-gluon exchange for the process under consideration. Let

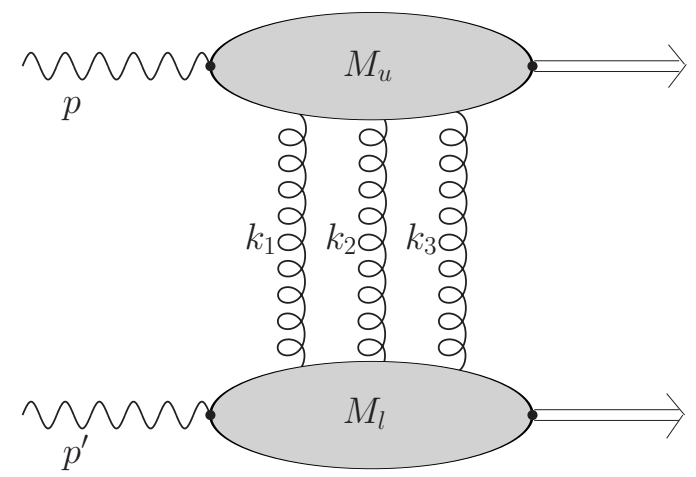

Figure 1: Diagrammatic representation of the scattering amplitude for a simple threegluon exchange process.

us first concentrate on the simple case in which the three gluons do not interact with each other, as illustrated in figure 1 We can write the corresponding amplitude as

$$
\mathcal{M}=\frac{i}{3 !} \int \frac{d^{4} k_{1}}{(2 \pi)^{4}} \frac{d^{4} k_{2}}{(2 \pi)^{4}} M_{u}^{\mu \nu \rho} \frac{\left(-\mathrm{i} g_{\mu \alpha}\right)}{k_{1}^{2}} \frac{\left(-i g_{\nu \beta}\right)}{k_{2}^{2}} \frac{\left(-i g_{\rho \gamma}\right)}{k_{3}^{2}} M_{l}^{\alpha \beta \gamma}
$$

where 3 ! is a combinatorial factor and $M_{u}^{\mu \nu \rho}$ and $M_{u}^{\mu \nu \rho}$ stand for the upper and lower shaded parts of the figure, respectively. These vertex functions also include the color factors and the contribution of the external particles.

Explicitly writing out the arguments of these functions for the case of incident photons, we have

$$
\begin{aligned}
& M_{u}^{\mu \nu \rho} \equiv M_{u}^{\mu \nu \rho}\left(p, \epsilon, k_{1}, k_{2}, k_{3}\right) \\
& M_{l}^{\alpha \beta \gamma} \equiv M_{l}^{\alpha \beta \gamma}\left(p^{\prime}, \epsilon^{\prime},-k_{1},-k_{2},-k_{3}\right) .
\end{aligned}
$$

The minus signs in front of the gluon momenta in the term $M_{l}^{\alpha \beta \gamma}$ arise because an incoming momentum in the upper vertex is treated as outgoing in the lower vertex and vice versa. The polarization vectors $\epsilon$ and $\epsilon^{\prime}$ of the incident photons will not be relevant in our discussion and will therefore not be written explicitly below. The three gluon momenta are related to the momentum transfer via $q=k_{1}+k_{2}+k_{3}$, and $q^{2}=t$.

Now we perform a Sudakov decomposition of the momenta by splitting each fourmomentum into its components parallel to the two light-like four-vectors $p$ and $p^{\prime}$ that have antiparallel three-momentum directions, and the remaining transverse part. For example, the vector $k_{1}$ is decomposed into

$$
k_{1}=\alpha_{1} p+\beta_{1} p^{\prime}+k_{1 T} .
$$


In high-energy collisions we can assume that the invariant masses are negligible compared to $s$. Hence $s=\left(p+p^{\prime}\right)^{2} \approx 2 p \cdot p^{\prime}$ and we can use the incident photon momenta for the decomposition also in the case of virtual photons. We will always denote by $\alpha$ the Sudakov component belonging to the upper incident momentum $p$, and by $\beta$ the one belonging to $p^{\prime}$. The integration measure can then be rewritten as

$$
d^{4} k_{i}=p \cdot p^{\prime} d \alpha_{i} d \beta_{i} d^{2} \mathbf{k}_{i}=\frac{1}{2} s d \alpha_{i} d \beta_{i} d^{2} \mathbf{k}_{i}, \quad i=1,2 .
$$

Furthermore, the transferred momenta are predominantly transverse (as was shown for the QED case in [46]), hence the denominator of a gluon propagator can be simplified to

$$
k_{i}^{2}=s \alpha_{i} \beta_{i}+k_{i T}^{2} \approx-\mathbf{k}_{i}^{2} .
$$

Also the metric tensor $g^{\mu \alpha}$ can be divided into longitudinal and transverse parts,

$$
g_{\mu \alpha}=\frac{2}{s}\left(p_{\mu} p_{\alpha}^{\prime}+p_{\alpha} p_{\mu}^{\prime}\right)+g_{\mu \alpha}^{T} .
$$

After the convolution with the vertex functions $M_{u}^{\mu \nu \rho}$ and $M_{l}^{\alpha \beta \gamma}$, the large contributions arise from the terms in which the momentum of the lower incident particle is contracted with the upper vertex, and vice versa. Phrased differently, one finds that the longitudinal gluon polarizations dominate in the high energy limit. We can therefore substitute

$$
g_{\mu \alpha} g_{\nu \beta} g_{\rho \gamma} \rightarrow \frac{8}{s^{3}} p_{\mu}^{\prime} p_{\nu}^{\prime} p_{\rho}^{\prime} p_{\alpha} p_{\beta} p_{\gamma},
$$

and obtain the amplitude in impact factor representation,

$$
\mathcal{M}=\frac{s}{3} \int \frac{d^{2} \mathbf{k}_{1}}{(2 \pi)^{2}} \frac{d^{2} \mathbf{k}_{2}}{(2 \pi)^{2}} \Phi_{u} \frac{1}{\mathbf{k}_{1}^{2}} \frac{1}{\mathbf{k}_{2}^{2}} \frac{1}{\mathbf{k}_{3}^{2}} \Phi_{l},
$$

with the impact factors being

$$
\begin{aligned}
\Phi_{u} & =\int \frac{d \beta_{1}}{2 \pi} \frac{d \beta_{2}}{2 \pi} M_{u}^{\mu \nu \rho} \frac{p_{\mu}^{\prime} p_{\nu}^{\prime} p_{\rho}^{\prime}}{s} \\
\Phi_{l} & =\int \frac{d \alpha_{1}}{2 \pi} \frac{d \alpha_{2}}{2 \pi} M_{l}^{\alpha \beta \gamma} \frac{p_{\alpha} p_{\beta} p_{\gamma}}{s} .
\end{aligned}
$$

The integrals can be disentangled here because the $\alpha$-parameters of the gluons can be neglected in $M_{u}^{\mu \nu \rho}$ (they are small compared to the $\alpha$-parameters of the quark lines), and likewise for the $\beta$-parameters in the lower vertex.

The above considerations are easily generalized to more sophisticated exchanges in which the $t$-channel gluons interact with each other as is shown in figure 2. Also here the dominant contributions come from the longitudinally polarized gluons and the resulting impact factors are exactly the same as above. The resulting amplitude can be written symbolically as

$$
\mathcal{M}=\frac{s}{3}\left\langle\Phi_{u}|\mathbf{G}| \Phi_{l}\right\rangle .
$$

In that general case the matrix element symbol stands for the integration over all undetermined momenta of the scattering amplitude - that is, $\mathbf{k}_{1}, \mathbf{k}_{2}, \mathbf{k}_{1}^{\prime}$ and $\mathbf{k}_{2}^{\prime}$ (the third gluon's momentum is fixed by the total momentum transfer). It also includes a 


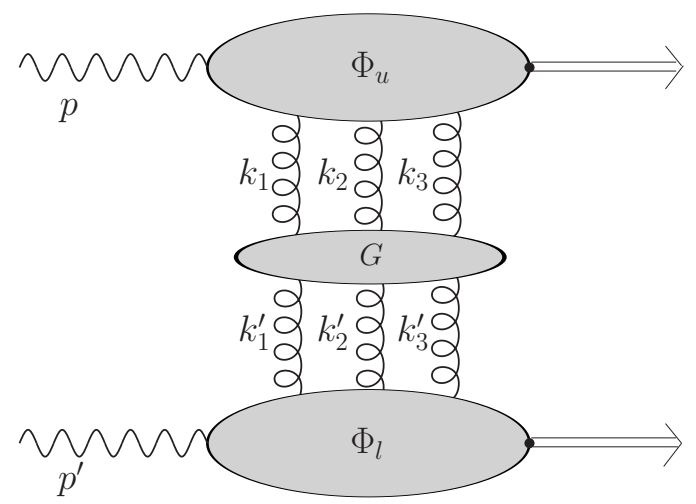

Figure 2: Impact factor representation of a scattering process with $t$-channel gluon interaction.

factor $(2 \pi)^{-4}$ as in the simple case discussed above. G denotes the Green function of the three-gluon exchange in transverse space. In this picture the simple three-gluon exchange process (fig. 1) is reproduced by setting

$$
\mathbf{G}=\frac{\delta^{2}\left(\mathbf{k}_{1}^{\prime}-\mathbf{k}_{1}\right) \delta^{2}\left(\mathbf{k}_{2}^{\prime}-\mathbf{k}_{2}\right)}{\mathbf{k}_{1}^{2} \mathbf{k}_{2}^{2} \mathbf{k}_{3}^{2}}
$$

It is also straightforward to generalize the derivation of the impact factors to exchanges with an arbitrary number of gluons in the $t$-channel. From the formulae above one can easily read off the factors and integrations which have to be associated with each additional gluon.

\subsection{Color algebra}

Let us now collect some basics of color algebra for the gauge group $\mathrm{SU}\left(N_{c}\right)$. Its Lie algebra has $N_{c}^{2}-1$ generators $t^{a}$ that satisfy the algebra

$$
\left[t^{a}, t^{b}\right]=i f_{a b c} t^{c}
$$

with the antisymmetric structure constants $f_{a b c}$. The anti-commutator of two generators defines the symmetric structure constants $d_{a b c}$,

$$
\left\{t^{a}, t^{b}\right\}=\frac{1}{N_{c}} \delta^{a b}+d_{a b c} t^{c} .
$$

Note that we write the structure constants with lower indices.

Sometimes we will use the so-called 'bird track' notation to illustrate color tensor contractions diagrammatically. The antisymmetric structure constants are drawn as a solid black circles and the color indices are written counterclockwise:

$$
f_{a b c}={ }_{b}^{a} .
$$

Then for example the nontrivial part of the color tensor of the $2 \rightarrow 3$ kernel in (8) is given by

$$
f_{b_{1} a_{1} k_{1}} f_{k_{1} a_{2} k_{2}} f_{k_{2} a_{3} b_{2}}=\mapsto \uparrow
$$


The color tensors for the integral kernels are drawn in such a way that the color indices $b_{i}$, which are to be contracted with the color labels of the amplitudes in the integral equations, are at the top of the diagram.

It is convenient to define the tensors

$$
\begin{aligned}
d^{a_{1} a_{2} \ldots a_{n}} & =\operatorname{Tr}\left(t^{a_{1}} t^{a_{2}} \ldots t^{a_{n}}\right)+\operatorname{Tr}\left(t^{a_{n}} t^{a_{n-1}} \ldots t^{a_{1}}\right), \\
f^{a_{1} a_{2} \ldots a_{n}} & =\frac{1}{i}\left[\operatorname{Tr}\left(t^{a_{1}} t^{a_{2}} \ldots t^{a_{n}}\right)-\operatorname{Tr}\left(t^{a_{n}} t^{a_{n-1}} \ldots t^{a_{1}}\right)\right] .
\end{aligned}
$$

Note that these definitions do not coincide with the structure constants for $n=3$. In fact, the structure constants are given in terms of these tensors by

$$
\begin{aligned}
d_{a_{1} a_{2} a_{3}} & =\frac{1}{2} d^{a_{1} a_{2} a_{3}}, \\
f_{a_{1} a_{2} a_{3}} & =\frac{1}{2} f^{a_{1} a_{2} a_{3}} .
\end{aligned}
$$

\subsection{The Odderon quark loop with three gluons}

Next we want to review some important properties of the $\gamma \rightarrow \eta_{c}$ impact factor with three gluons attached to it which was first calculated in [38. Three is the lowest possible number of gluons in the $t$-channel for which that impact factor exists.

The $\gamma \rightarrow \eta_{c}$ impact factor for three gluons consists of eight diagrams corresponding to the $2^{3}$ choices for the way in which the gluons couple to the the quark or antiquark. (Recall that due to the cuts applied to the amplitude the gluons do not cross each other.) One of these diagrams is shown in figure 3. Most of the details of the analytic

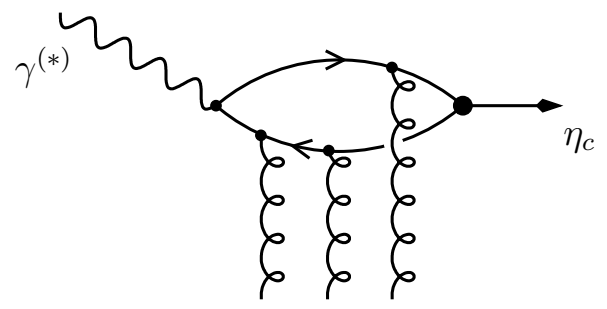

Figure 3: One of the diagrams contributing to the $\gamma \rightarrow \eta_{c}$ impact factor with three gluons.

expression for the impact factor will not be relevant for our considerations. A key property is that the Dirac structure of the coupling of the quark-antiquark pair to the $\eta_{c}$ meson is simply $\gamma^{5}$. The coefficient of the $\gamma^{5}$ factor can be related to the radiative two-photon width of the $\eta_{c}$, see 38. Further, it turns out that only transverse photon polarizations give a nonvanishing contribution to the impact factor. In the following we will often speak of the quark loop to which the Odderon is coupled. Unless otherwise stated this will always assume that there is a $\gamma_{\mu}$ matrix at the photon vertex and a $\gamma^{5}$ matrix at the meson vertex.

When calculating one particular diagram (out of the eight possible diagrams) one encounters five quark propagators of the form

$$
\frac{i(\not k+m)}{k^{2}-m^{2}},
$$


four additional $\gamma$ matrices (three gluon vertices and the photon vertex) and a $\gamma^{5}$ coming from the $\eta_{c}$ vertex. The gluon vertex $\gamma$ matrices get contracted with the light-like vector $p^{\prime}$ from the lower impact factor (see section 3.1). Using the fact that for light-like vectors $p$ the equation $\not p \not p=0$ holds, one easily reduces the expression in the $\gamma$ trace to a sum of terms that consist of maximally five $\gamma$ 's by permuting the gluon vertex $\gamma$ matrices next to each other.

When performing the trace for the case of massless quarks, one clearly gets zero because of the odd number of $\gamma$ matrices, and hence we need to take into account mass effects. That allows us to choose a mass term instead of a momentum term with a $\gamma$ matrix (in the numerator of the quark propagator $\not k+m$ ). In fact, only the case in which of the five propagator terms one mass and four momenta are combined gives a nonvanishing contribution. Let us stress this point: when calculating the impact factor, exactly one propagator term must lend its mass, the others their momenta with $\gamma$ matrices. Clearly, we will get a sum over the different possibilities of which propagator lends its mass, but for our purposes it will suffice to keep these considerations on a qualitative level.

Another important property is the behavior of an impact factor diagram under the exchange of its quark and antiquark lines. We can reassign the quark loop momenta in such a way that we get almost the same expression as before, apart from a minus sign for all propagator momenta in the $\gamma$ trace. For three gluons this means an overall relative plus sign because one propagator must give a mass instead of a momentum (as we have explained above) and the sign of the mass is not affected by the exchange. The set of all possible impact factor diagrams can be grouped into pairs in which the two diagrams are just related to each other by such an interchange of the quark and antiquark lines. Doing so, one reduces the number of diagrams that have to be calculated by two. This holds for the momentum and $\gamma$ part only, but the interchange of the quark lines naturally also reverses the order of the $S U\left(N_{c}\right)$ generators.

For three gluons we finally get four diagrams in this way, each coming together with two different traces of color matrices. Adding these two contributions one gets

$$
\operatorname{Tr}\left(t^{a_{3}} t^{a_{2}} t^{a_{1}}\right)+\operatorname{Tr}\left(t^{a_{1}} t^{a_{2}} t^{a_{3}}\right)=d^{a_{1} a_{2} a_{3}} .
$$

The full impact factor is then just the sum over the four different momentum diagrams together with the color tensor $d^{a_{1} a_{2} a_{3}}$ :

$$
\overbrace{F_{(3 ; 0)}}=d^{a_{1} a_{2} a_{3}}\left(\bar{\Pi}+\bar{\Pi}+\bar{\Pi}+\bar{\Pi}+\bar{\prod}\right) \equiv d^{a_{1} a_{2} a_{3}} F_{(3 ; 0)} .
$$

The schematic notation introduced here stands symbolically for the impact factor without color factors. The diagrams only show which gluons couple to the quark (upper line) and which to the antiquark (lower line) - the overall color tensor $d^{a_{1} a_{2} a_{3}}$ is extracted but the photon and $\eta_{c}$ vertices are implied. To obtain the final result, one needs to calculate these different diagrams.

As already said, we will not need the explicit form of the three-gluon impact factor. But there is one property that will be very important for our considerations, namely the symmetry under exchange of its momentum arguments. It does not hold for every contributing momentum diagram separately, but can only be seen after performing the sum over the different diagrams. 


\subsection{Mechanism of gluon number reduction}

Now we want to explain how an $n+1$ gluon diagram can be reduced to an $n$ gluon diagram. Clearly we can always find at least two gluons that are attached to the same quark line. Hence we can choose two which are separated only by one quark propagator. The additional factors that enter - compared to the $n$-gluon diagram - are a gluon vertex contracted with the incident momentum of the lower impact factor $p^{\prime}$ and the additional quark propagator between the two neighboring gluon vertices. The propagator again has a sum $\not k+m$ in its numerator but as the mass term is sandwiched between two factors of $\not p^{\prime}$ its contribution vanishes. Therefore, the addition of another gluon increases the number of $\gamma$ matrices by two. To reduce the number of $\gamma$ matrices in the trace, we use the same mechanism that we have already explained in the beginning of the previous section. Thereby we can always reduce the trace to one involving four $\gamma$ matrices, the $\gamma^{5}$ from the meson vertex, and one quark mass. In the following we want to have a closer look at this reduction mechanism and at the factors which occur. For that it will be important that we work with cut amplitudes, which amounts to putting the cut quark lines on-shell.

First we choose two gluons that are attached to the same quark line at neighboring vertices. Writing it in Feynman diagram notation, this part of the quark loop can be represented as

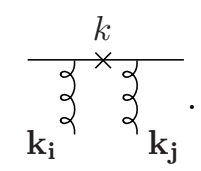

Let $k$ be the momentum of the quark line that lies between these two vertices. We will call the gluon further down along the line 'new' gluon, because we understand it as an additional gluon as compared to the $n$-gluon amplitude. According to the Cutkosky rules for calculating the discontinuity of the diagram, the 'new' quark propagator term is replaced according to

$$
\frac{i(\not k+m)}{k^{2}-m^{2}} \rightarrow i(\not k+m)\left[-2 \pi i \delta\left(k^{2}-m^{2}\right)\right]
$$

because a quark line between two neighboring gluons is always put on shell due to the cuts.

Let $j$ be the index of the new gluon. The new vertex gets contracted with the momentum of the lower incident particle $p^{\prime}$ to give $i g t^{a_{j}} \not p^{\prime}$. As was explained in section 3.1. for every gluon one also gets an integration over its Sudakov component parallel to $p^{\prime}$, i.e. over $\beta_{j}$. Putting it all together, one finds as additional factors apart from the new color trace:

$$
\begin{gathered}
\ldots \int \frac{d \beta_{j}}{2 \pi} i g \operatorname{Tr}\left(\ldots i(\not k+m) \not p^{\prime} \ldots\right)\left[-2 \pi i \delta\left(k^{2}-m^{2}\right)\right] \\
=\ldots i g \int d \beta_{j} \operatorname{Tr}\left(\ldots(\not k+m) \not p^{\prime} \ldots\right) \delta\left(k^{2}-m^{2}\right) .
\end{gathered}
$$

The neighboring gluon with momentum $\mathbf{k}_{i}$ in (33) also gives a $\not p^{\prime}$ in the $\gamma$-trace, and we can permute it with the quark line numerator and get a factor $2 p^{\prime} \cdot k$. By using the 
Sudakov decomposition for the quark line, $k=\alpha p+\beta p^{\prime}+k_{T}$, this can be absorbed by the $\delta$-function, and so we get

$$
i g \int d \beta_{j} \operatorname{Tr}\left(\ldots \not \not^{\prime} \ldots\right) \delta\left(\beta-\frac{\mathbf{k}^{2}+m^{2}}{\alpha s}\right) .
$$

We now shift the integration variables

$$
\begin{aligned}
& \beta_{i} \rightarrow \beta_{i}^{\prime}=\beta_{i}+\beta_{j}, \\
& \beta_{j} \rightarrow \beta_{j} .
\end{aligned}
$$

It is clear that all the $\beta$ parameters in the quark loop apart from the ones coming from the new quark propagator can be expressed in terms of $\beta_{i}^{\prime}$ and the $\beta$ parameters of the other gluons and of the quark loop momentum only, which means that the only occurrence of $\beta_{j}$ is in the new propagator. ${ }^{1}$ Now the integration over $\beta_{j}$ can be performed to cancel the $\delta$-function. In pictorial notation, we are left with the following identity:

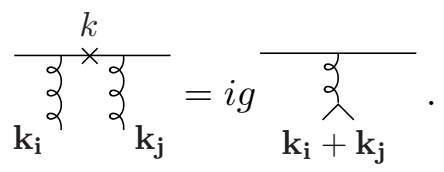

So far we have discussed only the momentum part of one particular diagram that contributes to the Odderon impact factor with $n+1$ gluons. We also have to consider the relative sign between two diagrams that are linked by interchange of the quark and antiquark lines. Compared to the $n$-gluon case we have included another quark propagator and a $\gamma$ matrix coming from the new gluon vertex. Independently of the number of attached gluons, always one mass term in the $\gamma$ trace has to be chosen, so every new propagator gives a new relative minus sign since the momentum term changes sign under the interchange of the fermion lines. (Note that this is due to the fact that we are calculating a closed fermion loop in which the direction of the momentum flow is reversed due to the interchange of the fermion lines.) Remembering that for 3 gluons we found a relative plus sign, we easily see that the relative sign between diagrams with interchanged quark and antiquark lines is $(-1)^{n+1}$. In other words, the momentum part of a diagram with an odd number of gluons is symmetric under the interchange of quark and antiquark, whereas an even number of gluons leads to an antisymmetric momentum part. Thus, we get $d$-type color tensors for odd numbers of attached gluons, $f$-type tensors for even numbers (with a factor of $i$ to cancel the $1 / i$ in the definition (27) of the $f$ tensors).

\subsection{The Odderon quark loop with four gluons}

We are now ready to express the $n+1$ gluon quark loop for the Odderon in terms of the one with $n$ gluons. The main calculation becomes clear in the step from three to four gluons. It is then easy to proceed to higher gluon numbers. The four-gluon impact factor consists of two color structures, each with four pairs of momentum diagrams. The terms in which all four gluons are attached to the same fermion line, for example,

\footnotetext{
${ }^{1}$ If one denotes the $\beta$ parameter of the incoming quark momentum at the left of (33) by $\tilde{\beta}$, one easily finds $\beta=\tilde{\beta}+\beta_{i}^{\prime}-\beta_{j}$, so one can see that $\beta_{j}$ in fact enters in the argument of the $\delta$-function.
} 
give the following structure (all traces are performed in the direction opposite to the quark lines):

$$
\operatorname{Tr}\left(t^{a_{4}} t^{a_{3}} t^{a_{2}} t^{a_{1}}-t^{a_{1}} t^{a_{2}} t^{a_{3}} t^{a_{4}}\right) \frac{\prod \prod}{\|\|}=-i f^{a_{1} a_{2} a_{3} a_{4}} \frac{\prod \|}{\|\|}
$$

where we have reversed the order of the color indices of the $f$ tensor, which gives an additional minus sign. For the complete four-gluon quark loop one finds the following diagrammatic representation:

$$
\begin{aligned}
& \overline{F_{(4 ; 0)}}=-i f^{a_{1} a_{2} a_{3} a_{4}}\left(\overline{\Pi \|}+\overline{\Pi \|}+\overline{\Pi \|}+\frac{\Pi \|}{\|\|}\right) \\
& -i f a_{3} a_{1} a_{2} a_{4}\left(\bar{\prod}+\bar{\Pi}+\bar{\Pi}+\bar{\prod}+\bar{\prod}+\bar{\prod}\right) \\
& \equiv-i f^{a_{1} a_{2} a_{3} a_{4}} F_{(4 ; 0)}^{(1)}-i f^{a_{3} a_{1} a_{2} a_{4}} F_{(4 ; 0)}^{(2)} \text {. }
\end{aligned}
$$

Clearly, there are many possible ways of reducing the single momentum diagrams to three-gluon diagrams along the lines of the previous section. But it is our aim to express it in terms of the full three-gluon impact factor $F_{(3 ; 0)}$. Motivated by the structure of the three-gluon $\gamma \rightarrow \gamma$ impact factor in the $C$-even channel [16] and by the general property of reggeization in the $n$-gluon amplitudes in that channel [24, 26] we now make an ansatz and show that it in fact gives the full impact factor. The following construction leads to the correct result for the four-gluon Odderon impact factor:

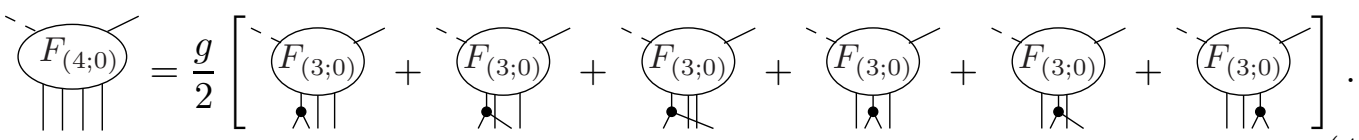

This diagrammatic notation symbolizes the contraction of the color tensor of the impact factor $F_{(3 ; 0)}$ with an $f_{a b c}$ tensor. In addition, one of the arguments of the amplitude $F_{(3 ; 0)}$ is actually the sum of two gluons' momenta. We will further abbreviate this expression containing sums of two arguments and contractions with a color tensor $f$ in the following way:

$$
\prod_{(4 ; 0)}=\frac{g}{2} \sum_{\substack{i, j \in\{1, \ldots, 4\} \\ i<j}}\left[\prod_{i} \prod_{j}\right] \star F_{(3 ; 0)}^{b_{1} b_{2} b_{3}}(i j)
$$

where $i$ and $j$ denote the position of the two 'merging' gluons in the four-gluon system and the same indices appear in the color tensor and in the sum of the momenta. The star symbolizes the contraction of the color tensor (drawn in bird track notation) with the tensor of the amplitude $F_{(3 ; 0)}^{b_{1} b_{2} b_{3}}$. The arguments of the function $F_{(3 ; 0)}$ are very much simplified here. Clearly, the momenta of all gluons enter as arguments of the function, but the main point on which we focus in this notation is that the momenta of the two merged gluons enter only with their sum. To shorten the notation, we simply write down the indices that correspond to the merged gluons. That notation means that, for example,

$$
F_{(3 ; 0)}^{b_{1} b_{2} b_{3}}(12)=F_{(3 ; 0)}^{b_{1} b_{2} b_{3}}(12,3,4)=F_{(3 ; 0)}^{b_{1} b_{2} b_{3}}\left(\mathbf{k}_{1}+\mathbf{k}_{2}, \mathbf{k}_{3}, \mathbf{k}_{4}\right)
$$


A string of indices stands for the sum of the corresponding momenta. As we have mentioned before, the three-gluon impact factor is symmetric under the exchange of its momentum arguments, so we do not need to worry about the position at which the merged gluons' sum enters as long as we choose it consistently in all the specific diagrams shown in (32). With this abbreviation of the momentum arguments, expression (42) has the following explicit form,

$$
\begin{aligned}
F_{(4 ; 0)}^{a_{1} a_{2} a_{3} a_{4}}=\frac{g}{2}\left[f_{a_{1} a_{2} k} F_{(3 ; 0)}^{k a_{3} a_{4}}(12)+f_{a_{1} a_{3} k} F_{(3 ; 0)}^{k a_{2} a_{4}}(13)+f_{a_{1} a_{4} k} F_{(3 ; 0)}^{k a_{2} a_{3}}(14)\right. \\
\\
\left.+f_{a_{2} a_{3} k} F_{(3 ; 0)}^{a_{1} k a_{4}}(23)+f_{a_{2} a_{4} k} F_{(3 ; 0)}^{a_{1} k a_{3}}(24)+f_{a_{3} a_{4} k} F_{(3 ; 0)}^{a_{1} a_{2} k}(34)\right] .
\end{aligned}
$$

To prove (42) one writes out all 24 diagrams of three-gluon quark loops according to (32). The momentum part of these diagrams is easy to handle. A pair of gluons that couple to a quark line together is just expanded to their respective positions in the four-gluon amplitude as shown in (39), corresponding to a factor $\frac{-i}{g}$. Two gluons that enter the antiquark line give an additional minus sign when expanded.

One might think that a problem arises when a gluon is sandwiched by two merged gluons as is the case for example in the second diagram on the right hand side of (42), because we have discussed only the case when two neighboring gluons at the same quark line reduce to one. Recall, however, that we can reduce all gluons that are attached to the same quark line to one. Only the sum of the momenta of the gluons then enters the amplitude. Therefore, we can use the mechanism described above also for the general case of any two gluons attached to the same quark line.

The color part is slightly more involved, because the $d^{b_{1} b_{2} b_{3}}$ tensor of the three-gluon quark loop gets contracted with the color tensor which is given in (43) in bird track notation. For example, if the first two gluons are merged, this tensor is $f_{b_{1} a_{1} a_{2}} \delta^{b_{2} a_{3}} \delta^{b_{3} a_{4}}$. In order to treat the tensors of this type we use the identity

$$
f_{a b k} d^{k c d}=f^{a b c d}-f^{b a c d}
$$

Applying it to all the color contractions and using the cyclic invariance of the $f$ tensors and the antisymmetry under reversal of all color indices, one ends up with three different tensors: $f^{a_{1} a_{2} a_{3} a_{4}}, f^{a_{3} a_{1} a_{2} a_{4}}$, and $f^{a_{1} a_{3} a_{2} a_{4}}$. If one now collects all diagrams that come with these respective color tensors, some contributions cancel (because the interchange of quark and antiquark line induces a sign change), others get a factor of 2 . The diagrams coming with the color tensor $f^{a_{1} a_{3} a_{2} a_{4}}$ cancel completely. We are then exactly left with the expression in (41), which confirms that our ansatz (42) was indeed correct.

In section 4 we will promote the ansatz of (42) to an ansatz for the full four-gluon amplitude $F_{4}$. However, for the future project of solving the integral equations in the $C$-odd channel with more than four gluons a different representation for the impact factor might also be useful. We therefore also want to give that form which does not make use of the explicit color tensor $d^{b_{1} b_{2} b_{3}}$ of the three-gluon impact factor. This is helpful because the quark loops for higher gluon numbers can be easily written in terms of the momentum part of the three gluon expression.

In order to obtain that representation we go back one step and write down the expression for the four-gluon quark loop in terms of only the momentum part $F_{(3 ; 0)}$ of 
the three-gluon amplitudes:

$$
\begin{aligned}
F_{(4 ; 0)}^{a_{1} a_{2} a_{3} a_{4}}=\frac{g}{2}\left\{f^{a_{1} a_{2} a_{3} a_{4}}\left[F_{(3 ; 0)}(12)-F_{(3 ; 0)}(14)+F_{(3 ; 0)}(23)+F_{(3 ; 0)}(34)\right]\right. \\
\\
\left.+f^{a_{3} a_{1} a_{2} a_{4}}\left[F_{(3 ; 0)}(12)-F_{(3 ; 0)}(13)+F_{(3 ; 0)}(24)-F_{(3 ; 0)}(34)\right]\right\} .
\end{aligned}
$$

Here we can identify the momentum parts corresponding to the two color tensors:

$$
\begin{aligned}
& F_{(4 ; 0)}^{(1)}=\frac{i g}{2}\left[F_{(3 ; 0)}(12)-F_{(3 ; 0)}(14)+F_{(3 ; 0)}(23)+F_{(3 ; 0)}(34)\right] \\
& F_{(4 ; 0)}^{(2)}=\frac{i g}{2}\left[F_{(3 ; 0)}(12)-F_{(3 ; 0)}(13)+F_{(3 ; 0)}(24)-F_{(3 ; 0)}(34)\right]
\end{aligned}
$$

By directly looking at (41) an even simpler representation of $F_{(4 ; 0)}^{(1)}$ can be found:

$$
F_{(4 ; 0)}^{(1)}=i g F_{(3 ; 0)}(23) .
$$

and the four-gluon quark loop is expressed as

$$
\begin{aligned}
F_{(4 ; 0)}^{a_{1} a_{2} a_{3} a_{4}}= & g f^{a_{1} a_{2} a_{3} a_{4}} F_{(3 ; 0)}(23) \\
& \left.+\frac{g}{2} f^{a_{3} a_{1} a_{2} a_{4}}\left[F_{(3 ; 0)}(12)-F_{(3 ; 0)}(13)+F_{(3 ; 0)}(24)-F_{(3 ; 0)}(34)\right]\right\} .
\end{aligned}
$$

In summary, we have derived two different (but equivalent) representations of the four-gluon quark loop in terms of the three-gluon quark loop: in the first representation (45) the three-gluon quark loop with its color structure is used, and that will be very convenient for the construction of the four-gluon Odderon amplitude in section 4 . The second representation (51) uses only the momentum part of the three-gluon expression and is useful for the investigation of the Odderon integral equations for more than four gluons.

\subsection{Generalization to an arbitrary number of gluons}

We now consider the general case of a quark loop with $n$ attached gluons, $n>3$. It consists of $2^{n}$ different diagrams. As could already be seen in the examples of three and four gluons in equations (32) and (41), four diagrams always lead to the same color structure, because switching the first or last gluon from the quark to the antiquark or vice versa clearly does not affect the color trace. We then get $2^{n-2}$ different color traces for the $n$-gluon quark loop. These diagrams differ in the relative attachment (to the quark or the antiquark) of the 'inner' $n-2$ gluons. In the previous sections we also grouped these $2^{n-2}$ different diagrams in pairs because the contributions of two diagrams in which quark and antiquark are interchanged differ only by a relative sign. For the purpose of the present section it will be more convenient to collect these pairs at the end.

In detail the combinatorics of the inner gluons is as follows. If all inner gluons couple to the quark line, one has the color trace $\operatorname{Tr}\left(t^{a_{n}} t^{a_{n-1}} \ldots t^{a_{1}}\right)$. Then there are $n-2$ different diagrams in which one inner gluon couples to the antiquark, whereas all others couple to the quark, and so on. For $k$ inner gluons attached to the antiquark and $n-2-k$ attached to the quark line, we get $\left(\begin{array}{c}n-2 \\ k\end{array}\right)$ different color traces. To obtain the 
correct color trace one simply has to write (in descending order of the gluon indices) the color matrices that correspond to the 'quark' gluons in front of the matrices of the 'antiquark' gluons (in ascending order). Summing all these contributions we get the complete set of contributions.

Now we have to take into account the pairing of diagrams with interchanged quark and antiquark lines. For example, the diagram in which only the first of the inner gluons couples to the antiquark line gives (apart from the relative sign and the reversed order of the color generators) the same result as the diagram in which all but the first inner gluon couple to the antiquark. We thus get $2^{n-3}$ color tensors of the type $d$ or $f$ together with four momentum diagrams each. We choose the convention to write down those diagrams of each pair where the first inner gluon is attached to the quark line, as we did also in the case of the diagrams of $F_{(4 ; 0)}^{(2)}$, see (41).

These momentum diagrams can now easily be expressed in terms of the two parts $F_{(4 ; 0)}^{(1)}$ and $F_{(4 ; 0)}^{(2)}$ of the four-gluon amplitude, see (48) and (49). If all inner gluons are attached to the same line, the resulting expression reads

$$
\begin{aligned}
-i f^{a_{1} \ldots a_{n}}(i g)^{n-3} F_{(3 ; 0)}(2 \ldots n-1) & \text { for even } n, \\
d^{a_{1} \ldots a_{n}}(i g)^{n-3} F_{(3 ; 0)}(2 \ldots n-1) & \text { for odd } n,
\end{aligned}
$$

where we have used (50) to express it in terms of $F_{(3 ; 0)}$. We have also reversed the order of the color indices so that the gluons that are attached to the quark line are written in ascending order. This introduces a minus sign for all $f$ tensors.

If at least one of the inner gluons couples to the antiquark, the structure naturally is more complicated. We will explain the construction of the general form, but also give the example of five gluons along the way.

First one has to identify all $2^{n-3}-1$ additional combinations of how the inner gluons can couple to the quark or antiquark lines. For five gluons these are

$$
d^{a_{4} a_{3} a_{1} a_{2} a_{5} \prod_{\prod}, \quad d^{a_{3} a_{1} a_{2} a_{4} a_{5}} \prod_{\|}, \quad d^{a_{4} a_{1} a_{2} a_{3} a_{5}} \prod_{\|} .}
$$

We only show the coupling of the inner gluons here, because the first and the last gluon do not affect the color structure as was explained above. Symbolically, we therefore draw the outer gluons as dashed lines and do not specify to which line they couple.

Let $i_{1}, \ldots, i_{k}$ now be the indices of the inner gluons that are attached to the antiquark line and $j_{1}, \ldots, j_{n-2-k}$ the indices of the inner gluons attached to the quark line. As explained before, the merging of two gluons at the antiquark line introduces an additional minus sign. Now we can write down the complete expression for one particular color structure,

$$
\begin{aligned}
-i(-1)^{k-1} f^{a_{i_{k}} \ldots a_{i_{1}} a_{1} a_{j_{1}} \ldots a_{j_{n-2-k}} a_{n}}(i g)^{n-4} F_{(4 ; 0)}^{(2)}\left(1, j_{1} \ldots j_{n-2-k}, i_{1} \ldots i_{k}, n\right) & \text { for even } n, \\
\quad(-1)^{k-1} d^{a_{i_{k}} \ldots a_{i_{1}} a_{1} a_{j_{1}} \ldots a_{j_{n-2-k}} a_{n}}(i g)^{n-4} F_{(4 ; 0)}^{(2)}\left(1, j_{1} \ldots j_{n-2-k}, i_{1} \ldots i_{k}, n\right) & \text { for odd } n .
\end{aligned}
$$

In order to avoid confusion here we have reinstated all four arguments of the amplitude $F_{(4 ; 0)}^{(2)}$, and again the collection of indices in one argument stands for the sum of the respective gluons' momenta. Adding all contributions, one arrives at the expression for the general $n$-gluon quark loop in terms of three- and four-gluon quark loop amplitudes. 
As an example of this construction, the explicit form for the full impact factor for five gluons is

$$
\begin{aligned}
F_{(5 ; 0)}= & (i g)^{2} d^{a_{1} a_{2} a_{3} a_{4} a_{5}} F_{(3 ; 0)}(1,234,5)-i g d^{a_{4} a_{3} a_{1} a_{2} a_{5}} F_{(4 ; 0)}^{(2)}(1,2,34,5) \\
& +i g d^{a_{3} a_{1} a_{2} a_{4} a_{5}} F_{(4 ; 0)}^{(2)}(1,24,3,5)+i g d^{a_{4} a_{1} a_{2} a_{3} a_{5}} F_{(4 ; 0)}^{(2)}(1,23,4,5) .
\end{aligned}
$$

Finally, one can use (49) to express all the parts in terms of the three-gluon amplitude $F_{(3 ; 0)}$ only. As we have chosen the diagrams in such a way that the second gluon is attached to the quark line, the sum of quark line gluons enters the amplitude $F_{(4 ; 0)}$ as the second argument and the sum of antiquark line gluons enters as the third argument. We thus arrive at the expression for the different color parts of the $n$-gluon quark loop in terms of the three-gluon quark loop $F_{(3 ; 0)}$. For even $n$ it reads:

$$
\begin{aligned}
-i \frac{(-1)^{k-1}}{2}(i g)^{n-3} & f^{a_{i_{k}} \ldots a_{i_{1}} a_{1} a_{j_{1}} \ldots a_{j_{n-2-k}} a_{n}} \\
& {\left[F_{(3 ; 0)}\left(1 j_{1} \ldots j_{n-2-k}, i_{1} \ldots i_{k}, n\right)-F_{(3 ; 0)}\left(1 i_{1} \ldots i_{k}, j_{1} \ldots j_{n-2-k}, n\right)\right.} \\
+ & \left.F_{(3 ; 0)}\left(1, j_{1} \ldots j_{n-2-k} n, i_{1} \ldots i_{k}\right)-F_{(3 ; 0)}\left(1, j_{1} \ldots j_{n-2-k}, i_{1} \ldots i_{k} n\right)\right] .
\end{aligned}
$$

For odd $n$, once again the $f$ color tensor has to be replaced by a $d$ tensor and the factor $-i$ has to be dropped. To get the full impact factor, all $2^{n-3}$ contributions have to be added.

Again, we want to give the final expression for the five-gluon case to illustrate our results:

$$
\begin{aligned}
F_{(5 ; 0)}=- & g^{2} d^{a_{1} a_{2} a_{3} a_{4} a_{5}} F_{(3 ; 0)}(1,234,5) \\
+\frac{g^{2}}{2} d^{a_{4} a_{3} a_{1} a_{2} a_{5}}[ & F_{(3 ; 0)}(12,34,5)-F_{(3 ; 0)}(134,2,5)+F_{(3 ; 0)}(1,25,34) \\
& \left.-F_{(3 ; 0)}(1,2,345)\right] \\
-\frac{g^{2}}{2} d^{a_{3} a_{1} a_{2} a_{4} a_{5}} & F_{(3 ; 0)}(124,3,5)-F_{(3 ; 0)}(13,24,5)+F_{(3 ; 0)}(1,245,3) \\
& \left.-F_{(3 ; 0)}(1,24,35)\right] \\
-\frac{g^{2}}{2} d^{a_{4} a_{1} a_{2} a_{3} a_{5}} & F_{(3 ; 0)}(123,4,5)-F_{(3 ; 0)}(14,23,5)+F_{(3 ; 0)}(1,235,4) \\
& \left.-F_{(3 ; 0)}(1,23,45)\right] .
\end{aligned}
$$

In summary, we have computed the $\gamma \rightarrow \eta_{c}$ impact factor for an arbitrary number of gluons. We have shown how it can be expressed in terms of the three-gluon impact factor. For each $n$ the impact factor is a superposition of terms in which in the momentum part a subset of the gluons behaves like a single gluon in the three-gluon impact factor, that is only the sum of their momenta enters.

In appendix $\mathrm{A}$ we discuss how the construction of the $n$-gluon quark loop can be performed for the $\gamma \rightarrow \gamma$ impact factor, that is in the $C$-even sector, in a completely analogous way. 


\section{Solution for the four-gluon Odderon amplitude}

In [17, 18, it was observed that the three-gluon amplitude $D_{3}$ in the EGLLA is a superposition of two gluon amplitudes $D_{2}$,

$$
D_{3}^{a_{1} a_{2} a_{3}}\left(\mathbf{k}_{1}, \mathbf{k}_{2}, \mathbf{k}_{3}\right)=\frac{g}{2} f_{a_{1} a_{2} a_{3}}\left[D_{2}(12,3)-D_{2}(13,2)+D_{2}(1,23)\right] .
$$

This result required that the impact factor $D_{(3 ; 0)}$ describing the $\gamma^{*} \rightarrow \gamma^{*}$ transition with three $t$-channel gluons could be expressed in terms of the two-gluon impact factor $D_{(2 ; 0)}$, see (79). A similar observation was made in a part of the five-gluon state in the $C$-even sector, namely in the part containing the two-to-four gluon transition vertex, see [24]. In both cases the solution is given as a superposition of lower amplitudes in each of which a pair of gluons combines into a single gluon. In 26] this reggeization of the gluon was discussed also for more than two gluons which combine into one. A remarkable property of of the EGLLA in the $C$-even sector is that reggeization leads to exact solutions of the integral equations for all odd numbers of gluons. That means that the amplitudes $D_{2 l+1}$ can then be expressed in terms of the amplitudes $D_{2}, D_{4}, \ldots, D_{2 l}$. This was found explicitly for up to six gluons and argued to hold also for general $l$ in 24.

Motivated by these observations in the $C$-even channel we now construct a fourgluon amplitude for the $C$-odd channel in the same spirit. More precisely, we will show that the following ansatz solves the integral equation (5) for the full four-gluon state $F_{4}$ :

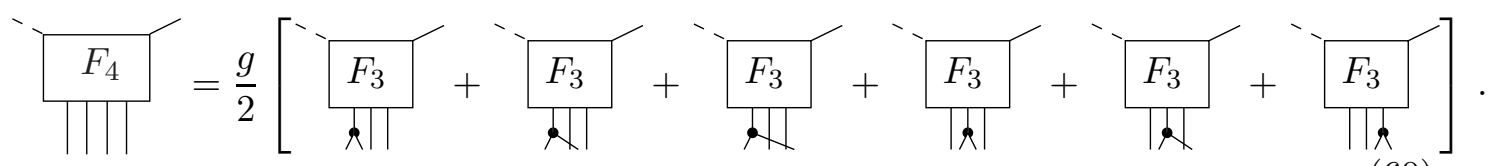

These $F_{3}$-diagrams will be called 'splitting pairs' from now on. The amplitudes $F_{3}$ again have three arguments, one of which is the sum of the two merged gluons. Again, a contraction of the color tensor of the amplitude $F_{3}^{b_{1} b_{2} b_{3}}$ with the structure constant $f$ is implied. Writing out the ansatz explicitly, we have

$$
\begin{aligned}
F_{4}^{a_{1} a_{2} a_{3} a_{4}}= & \frac{g}{2}\left[f_{a_{1} a_{2} k} F_{3}^{k a_{3} a_{4}}(12,3,4)+f_{a_{1} a_{3} k} F_{3}^{k a_{2} a_{4}}(13,2,4)+f_{a_{1} a_{4} k} F_{3}^{k a_{2} a_{3}}(14,2,3)\right. \\
& \left.+f_{a_{2} a_{3} k} F_{3}^{a_{1} k a_{4}}(1,23,4)+f_{a_{2} a_{4} k} F_{3}^{a_{1} k a_{3}}(1,24,3)+f_{a_{3} a_{4} k} F_{3}^{a_{1} a_{2} k}(1,2,34)\right] .
\end{aligned}
$$

Note that in this ansatz the full amplitude $F_{4}$ is expressed in terms of $F_{3}$ in exactly the same way as in our result (42) the four-gluon impact factor $F_{(4 ; 0)}$ was given in terms of the three-gluon impact factor $F_{(3 ; 0)}$.

The above ansatz already leads to a very simple cancellation in the four-gluon Odderon equation (6). One expresses all amplitudes $F_{4}$ and the quark loop $F_{(4 ; 0)}$ in terms of three-gluon diagrams. Then one can use the BKP equation (11) to evaluate the term $\omega F_{4}^{a_{1} a_{2} a_{3} a_{4}}$ and obtains exactly the same expression for the quark loop as on the right hand side of the equation. Thus, one is left with an equation in which only the full three-gluon amplitude $F_{3}$ appears.

We now consider one particular splitting pair of gluons. When using the BKP equation to cancel the $\omega$-term and the quark loop, one gets additional terms involving the 
three-gluon amplitude $F_{3}$ in which the corrections due to the kernel or the $\beta$ function occur before the splitting of one gluon into two. There are three virtual $(\beta)$ corrections of this kind: two where the $\beta$-term acts on a gluon that does not split and one where it acts on the gluon that further down splits into two gluons. In the four-gluon equation there are four virtual corrections. Two of these act on gluons coming directly from the amplitude $F_{4}$, i. e. that have not emerged from a splitting. These cancel the corresponding two terms coming from the three-gluon equation. The other two virtual corrections involve the two gluons that have emerged from the gluon splitting. One then easily finds that these terms together with the upper correction of the splitting gluon exactly cancel the contribution of the $2 \rightarrow 2$ kernel acting on the two gluons that have emerged from the splitting.

This means that all the virtual corrections and the term in which the splitting and the kernel take place in the same two gluons have canceled. We have considered only one particular pair of splitting gluons but the cancellation immediately extends to the sum of all splittings. Now one is left exactly with all the terms involving the $2 \rightarrow 3$ kernel and the remaining $2 \rightarrow 2$ terms.

At this stage one can collect all the diagrams in which one particular gluon, say the $i$ th outgoing gluon, passes the kernel and the splitting without being affected. Then one is left with four groups of identical expressions that cancel separately due to an identity that was already discussed in the context of reggeization in the Pomeron in 24, and in a somewhat different exposition also in 19]. We show this identity in pictorial language and omit the unaffected gluon:

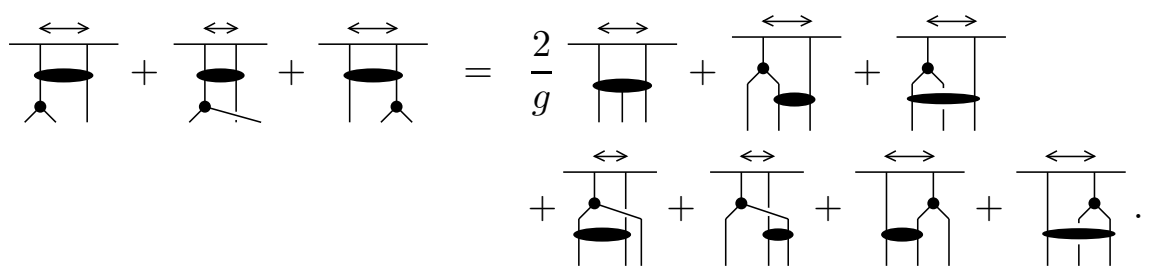

The arrows on top of the diagrams symbolize the symmetry of the amplitude under simultaneous exchange of momentum and color labels. In our case the interactions mediated by the integral kernel get contracted with the three gluon Odderon amplitude which also exhibits this symmetry as we have explained earlier. That symmetry is the only property of the amplitude that is needed for the proof of this identity. That completes our proof that the ansatz (60) for $F_{4}$ in fact solves the full integral equation (5) for the four-gluon Odderon state in the EGLLA.

Our result establishes that gluon reggeization takes place in the $C$-odd channel in the same way as in the $C$-even channel. At the same time, it shows that one cannot couple an actual four-gluon state to the $\gamma \rightarrow \eta_{c}$ impact factor in EGLLA. Instead, the impact factor couples only to a superposition of three-gluon Odderon states.

An important property of our solution for $F_{4}$ is that it satisfies the same Ward-type identities which were found for the $n$-gluon amplitudes in the $C$-even sector in [26]. These identities follow directly from (60) and from the fact that $F_{3}$ vanishes if one of the three gluons carries zero transverse momentum. More precisely, we find that $F_{4}$ vanishes if the first or last gluon momentum is zero,

$$
\left.F_{4}\left(\mathbf{k}_{1}, \mathbf{k}_{2}, \mathbf{k}_{3}, \mathbf{k}_{4}\right)\right|_{\mathbf{k}_{1}=0}=\left.F_{4}\left(\mathbf{k}_{1}, \mathbf{k}_{2}, \mathbf{k}_{3}, \mathbf{k}_{4}\right)\right|_{\mathbf{k}_{4}=0}=0
$$


whereas for the case that the inner gluon's momenta vanish the amplitude reduces to the lower amplitude $F_{3}$. The crucial point here is the specific behavior of the color tensors in this reduction. For the case $\mathbf{k}_{2}=0$ we find

$$
\left.F_{4}^{a_{1} a_{2} a_{3} a_{4}}\left(\mathbf{k}_{1}, \mathbf{k}_{2}, \mathbf{k}_{3}, \mathbf{k}_{4}\right)\right|_{\mathbf{k}_{2}=0}=g f_{a_{1} a_{2} k} F_{3}^{k a_{3} a_{4}}\left(\mathbf{k}_{1}, \mathbf{k}_{3}, \mathbf{k}_{4}\right),
$$

or alternatively

$$
\left.F_{4}^{a_{1} a_{2} a_{3} a_{4}}\left(\mathbf{k}_{1}, \mathbf{k}_{2}, \mathbf{k}_{3}, \mathbf{k}_{4}\right)\right|_{\mathbf{k}_{2}=0}=g f_{a_{2} a_{3} k} F_{3}^{a_{1} k a_{4}}\left(\mathbf{k}_{1}, \mathbf{k}_{3}, \mathbf{k}_{4}\right)+g f_{a_{2} a_{4} k} F_{3}^{a_{1} a_{3} k}\left(\mathbf{k}_{1}, \mathbf{k}_{3}, \mathbf{k}_{4}\right) .
$$

To obtain the last two identities we have made use of the overall color neutrality of the four gluons. Similarly, we find for the case $\mathbf{k}_{3}=0$

$$
\left.F_{4}^{a_{1} a_{2} a_{3} a_{4}}\left(\mathbf{k}_{1}, \mathbf{k}_{2}, \mathbf{k}_{3}, \mathbf{k}_{4}\right)\right|_{\mathbf{k}_{3}=0}=g f_{k a_{3} a_{4}} F_{3}^{a_{1} a_{2} k}\left(\mathbf{k}_{1}, \mathbf{k}_{2}, \mathbf{k}_{4}\right),
$$

which also equals

$$
\left.F_{4}^{a_{1} a_{2} a_{3} a_{4}}\left(\mathbf{k}_{1}, \mathbf{k}_{2}, \mathbf{k}_{3}, \mathbf{k}_{4}\right)\right|_{\mathbf{k}_{3}=0}=g f_{a_{2} a_{3} k} F_{3}^{a_{1} k a_{4}}\left(\mathbf{k}_{1}, \mathbf{k}_{2}, \mathbf{k}_{4}\right)+g f_{a_{1} a_{3} k} F_{3}^{k a_{2} a_{4}}\left(\mathbf{k}_{1}, \mathbf{k}_{2}, \mathbf{k}_{4}\right) .
$$

These are exactly the same identities that also hold for the reggeizing part of the fourgluon amplitude $D_{4}$ in the $C$-even channel, that is for the part which does not contain the two-to-four gluon vertex. For a detailed discussion of the Ward-type identities and of their significance for finding an effective field theory of interacting reggeized gluons we refer the reader to [26].

Note that up to now we have obtained our results only for the BLV Odderon solution 34. This is because we have considered the integral equations of the EGLLA for the case of the $\gamma \rightarrow \eta_{c}$ impact factor, and the BLV solution is the only known Odderon solution which couples to that impact factor in leading order. In the next section we will show that an analogous solution for the four-gluon amplitude exists also for the Janik-Wosiek (JW) Odderon solution [33.

\section{Generalization to other Odderon solutions}

The BLV solution that we have discussed so far is not the only possible solution of the BKP equation for the $C$-odd three-gluon state. The other class of solutions has the characteristic property that it vanishes if two of the transverse gluon coordinates coincide. That class comprises the Janik-Wosiek Odderon solution [33, and we will refer to that class generically as the JW Odderon. In order to study that class of solutions in the EGLLA we have to make a different choice for the inhomogeneous term of the integral equations that we have discussed in section 2 . In order to couple the JW Odderon to an impact factor in leading order we should consider an impact factor in which the three gluons are coupled to three different partons, i. e. we need three different points in transverse space to couple the gluons to. A suitable choice is a baryonic impact factor. The use of perturbation theory is in general questionable for light baryons. For the purpose of the present paper, however, we can think of a heavy baryon or of a large momentum transfer $\sqrt{t}$ in the case of a light baryon. Note that the integral equations of section 2 remain fully valid after replacing the $\gamma \rightarrow \eta_{c}$ impact factors $F_{(n ; 0)}$ by suitable baryonic impact factors. 
In contrast to the photon and the $\eta_{c}$ meson baryons are not eigenstates of $C$-parity. Baryonic impact factors therefore contain both $C$-even and $C$-odd contributions. A full discussion of the baryonic impact factor with arbitrary numbers of gluons is beyond the scope of the present paper. Instead, we will restrict ourselves to the impact factor relevant for the JW solution and for up to four gluons. We hence consider only diagrams in which at least one gluon is attached to each of the three quarks in the baryon, since all other diagrams would vanish when folded with the JW solution. The diagrams we have to consider for three gluons are hence of the type (to be specific here we choose a $p \rightarrow p$ impact factor)

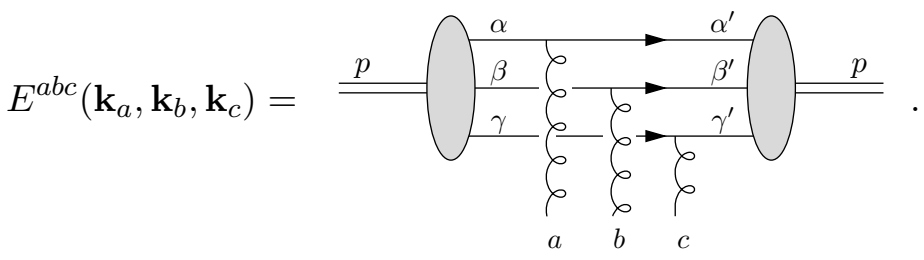

Incidentally, this diagram contributes only to the $C$-odd channel. This is most easily seen from its color factor,

$$
\varepsilon_{\alpha \beta \gamma} t_{\alpha^{\prime} \alpha}^{a} t_{\beta^{\prime} \beta}^{b} t_{\gamma^{\prime} \gamma}^{c} \varepsilon_{\alpha^{\prime} \beta^{\prime} \gamma^{\prime}}=\frac{1}{2} d_{a b c}
$$

which contains only the symmetric color configuration of the three gluons. The full relevant impact factor $B_{(3 ; 0)}^{p \rightarrow p} J W$ is obtained by adding all diagrams of this type. (For a baryon with three different constituent quark flavors we have six diagrams, otherwise it can be less with suitable symmetry factors for equal quark flavors.) After this summation the impact factor is symmetric in the color labels and in the momenta of the three gluons separately. Since our diagrams contribute only to the $C$-odd channel it is clear that the corresponding diagrams for the $\bar{p} \rightarrow \bar{p}$ impact factor have the opposite sign, but are otherwise identical. This is again due to the sign change of the vertices when replacing all quarks by antiquarks. Therefore the $C$-odd impact factor for the JW Odderon solution is identical to the $p \rightarrow p$ impact factor in this case,

$$
B_{(3 ; 0)}^{\mathcal{O} J W}=\frac{1}{2}\left[B_{(3 ; 0)}^{p \rightarrow p J W}-B_{(3 ; 0)}^{\bar{p} \rightarrow \bar{p} J W}\right]=B_{(3 ; 0)}^{p \rightarrow p J W} .
$$

As in the case of the $\gamma \rightarrow \eta_{c}$ impact factor, we will not need the specific dependence of the diagram $E$ in (68) on the gluon momenta, for phenomenological models we refer the reader to [48 and [49] (see also [50]). Instead, we will again make use of the reduction mechanism explained in section 3.4 and express the relevant diagrams with four gluons in terms of three-gluon diagrams of the type (68). Note that the same cuts are applied to the amplitudes as before such that the quarks between the gluons are set on-shell. Therefore all conditions for the reduction mechanism are fulfilled and we can apply it to each pair of gluons coupled to the same quark line.

Let us now consider the baryonic impact factor with four gluons. Given the two classes of Odderon solutions with three gluons it is natural to expect that there will also be two distinct classes of solutions in the case of four gluons. Here we concentrate only on the JW type solution, and in analogy to the three-gluon case we consider only diagrams contributing to the impact factor in which each quark has at least one gluon 
coupled to it. In the case of four gluons the resulting diagrams still contain both $C$ even and $C$-odd contributions. We therefore need to make a projection onto the $C$-odd amplitude by taking one half of the difference between the baryonic impact factor and its anti-baryonic analog.

Obviously, in all possible diagrams there is exactly one quark to which two gluons are attached. We hence have a sum over all six possible pairs of gluons. As an example we consider the first two gluons being coupled to the same quark. A typical diagram is

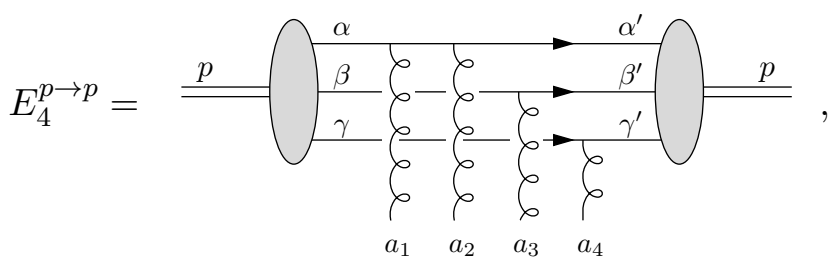

and we have to sum over all possible permutations of the quark lines exactly as in the three-gluon impact factor. The color part of that diagram is

$$
\varepsilon_{\alpha \beta \gamma}\left(t^{a_{2}} t^{a_{1}}\right)_{\alpha^{\prime} \alpha} t_{\beta^{\prime} \beta^{3}}^{a_{3}} t_{\gamma^{\prime} \gamma}^{a_{4}} \varepsilon_{\alpha^{\prime} \beta^{\prime} \gamma^{\prime}}
$$

since the three quarks are in an antisymmetric color state in the baryon.

In the case of the impact factor with an anti-baryon, the possible diagrams are obtained in the same way. The diagram corresponding to the one above is

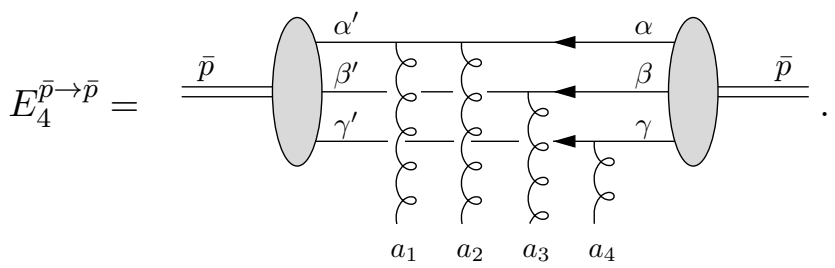

For later convenience we have already relabeled the summation indices for the colors in the incoming and outgoing baryon. The color factor for this diagram is

$$
\varepsilon_{\alpha^{\prime} \beta^{\prime} \gamma^{\prime}}\left(t^{a_{1}} t^{a_{2}}\right)_{\alpha^{\prime} \alpha} t_{\beta^{\prime} \beta}^{a_{3}} t_{\gamma^{\prime} \gamma}^{a_{4}} \varepsilon_{\alpha \beta \gamma} .
$$

The momentum parts of both diagrams are identical, in particular there is no sign change (in contrast to the three-gluon diagram, see above) because we have an even number of gluons here. We can now apply the reduction formula (39) and find that the momentum part of both diagrams is $i g$ times the momentum part of the three-gluon diagram $E$ of (68) with the three momentum arguments $\left(\mathbf{k}_{1}+\mathbf{k}_{2}\right), \mathbf{k}_{3}$, and $\mathbf{k}_{4}$. The difference of the color factors of the two diagrams, on the other hand, is

$$
\frac{1}{2} \varepsilon_{\alpha \beta \gamma}\left(t^{a_{2}} t^{a_{1}}-t^{a_{1}} t^{a_{2}}\right)_{\alpha^{\prime} \alpha} t_{\beta^{\prime} \beta}^{a_{3}} t_{\gamma^{\prime} \gamma}^{a_{4}} \varepsilon_{\alpha^{\prime} \beta^{\prime} \gamma^{\prime}}=-\frac{1}{2} i f_{a_{1} a_{2} k} \varepsilon_{\alpha \beta \gamma} t_{\alpha^{\prime} \alpha}^{k} t_{\beta^{\prime} \beta}^{a_{3}} t_{\gamma^{\prime} \gamma}^{a_{4}} \varepsilon_{\alpha^{\prime} \beta^{\prime} \gamma^{\prime}},
$$

where we have expressed that difference in terms of the color factor (69) of the threegluon diagram $E$.

Hence the total contribution of our two diagrams to the $C$-odd channel is

$$
\begin{aligned}
& \frac{1}{2}\left[\left(E_{4}^{p \rightarrow p}\right)^{a_{1} a_{2} a_{3} a_{4}}\left(\mathbf{k}_{1}, \mathbf{k}_{2}, \mathbf{k}_{3}, \mathbf{k}_{4}\right)-\left(E_{4}^{\bar{p} \rightarrow \bar{p}}\right)^{a_{1} a_{2} a_{3} a_{4}}\left(\mathbf{k}_{1}, \mathbf{k}_{2}, \mathbf{k}_{3}, \mathbf{k}_{4}\right)\right]= \\
& =\frac{1}{2} g f_{a_{1} a_{2} k} E^{k a_{3} a_{4}}\left(\mathbf{k}_{1}+\mathbf{k}_{2}, \mathbf{k}_{3}, \mathbf{k}_{4}\right) .
\end{aligned}
$$


The same mechanism obviously holds for the other permutations of the quark (respectively antiquark) lines, and the last formula then naturally extends to the sum of those diagrams. It remains to sum over all pairs of gluons, and for each pair of gluons we obtain an analogous expression. Denoting the full impact factor obtained in this way by $B_{(4 ; 0)}^{\mathcal{O} J W}$ we can write it in terms of the corresponding three-gluon impact factor $B_{(3 ; 0)}^{\mathcal{O} J W}$ as

$$
B_{(4 ; 0)}^{\mathcal{O} J W}=\frac{g}{2} \sum_{\substack{i, j \in\{1, \ldots, 4\} \\ i<j}}\left[\prod_{i}\right\rfloor_{j} \| \star\left(B_{(3 ; 0)}^{\mathcal{O} J W}\right)^{b_{1} b_{2} b_{3}}(i j),
$$

where we have again used the notation introduced in section 3.5. We recall that the superscript $J W$ refers to the fact that we have restricted our analysis to diagrams in which the gluons are coupled to all three quarks, that is to three distinct points in transverse space, hence giving rise to the JW Odderon solution. Note that this formula is identical to the way (43) in which the $\gamma \rightarrow \eta_{c}$ impact factor with four gluons was expressed in terms of the one with three gluons. Clearly, reggeization takes place in the same way in both impact factors.

Let us now proceed to the full three- and four-gluon amplitudes arising from the part of the baryonic impact factor relevant to the JW Odderon. The coupled integral equations for these amplitudes $B_{3}^{\mathcal{O} J W}$ and $B_{4}^{\mathcal{O} J W}$ are completely analogous to those for $F_{3}$ and $F_{4}$, see (11) and (5), with the $\gamma \rightarrow \eta_{c}$ impact factors replaced by the impact factors $B_{(3 ; 0)}^{\mathcal{O} J W}$ and $B_{(4 ; 0)}^{\mathcal{O} J W}$ discussed above. Since the latter two impact factors are related to each other in exactly the same way as the three- and four-gluon $\gamma \rightarrow \eta_{c}$ impact factors we can immediately apply the result of section 4 . Hence we find a solution for the full four-gluon amplitude $B_{4}^{\mathcal{O} J W}$ as a superposition of three-gluon amplitudes $B_{3}^{\mathcal{O} J W}$ in exactly the way given in (60) or in (61). We conclude that also in the JW class of Odderon solutions an actual four-gluon state does not contribute to the amplitude. Instead, due to reggeization the four-gluon amplitude has the analytic properties induced by the corresponding three-gluon amplitude. Again, reggeization takes place in the same way as in the $C$-even sector. Also here the Ward-type identities of [26] are fulfilled.

Two more remarks are in order concerning the baryonic impact factor and the way in which we have computed it. In the present section we have obtained the $C$-odd contribution to the impact factors as the difference of the baryonic impact factor and its $C$-conjugate, that is by explicitly projecting onto the $C$-odd channel. That method was also used (in position space) in [35] where the BLV Odderon solution was found in the dipole picture. Implicitly, we have done something similar in the calculation of the quark loop in the $\gamma \rightarrow \eta_{c}$ impact factor in section 3 above. There it was the $\gamma^{5}$ matrix in the loop which caused the relative signs between two diagrams related to each other by the exchange of quark and antiquark line (corresponding to a $C$-parity transformation of the quark-antiquark intermediate states). We found it more convenient to stay as close as possible to previous calculations of the $\gamma \rightarrow \eta_{c}$ impact factor with three gluons. Baryonic impact factors always contain both $C$-even and $C$-odd contributions and therefore in this case the explicit projection is needed, in contrast to the $\gamma \rightarrow \eta_{c}$ impact factor which couples only to $C$-odd states of gluons.

The calculation of the full baryonic impact factors for arbitrary numbers of gluons is clearly feasible with the methods presented here, both for $C$-even and for $C$-odd 
states of gluons. The calculation of those impact factors would be important for a better understanding of reggeization of the gluon in the high energy limit. We expect that taking into account also contributions in which not all quark lines have gluons attached one would obtain couplings to both classes of Odderon solution, that is JW and BLV solutions. It should then be possible to obtain full solutions of the integral equations for four gluons as superpositions of full JW and BLV solutions in analogy to the solutions found here. That would provide further evidence for the universality of gluon reggeization at high energies.

\section{Summary and outlook}

In this paper we have made the first step in a systematic investigation of $C$-odd exchanges in the color glass condensate. We have used the EGLLA, that is the perturbative approach based on the resummation of logarithms of the energy where we allow the number of gluons to fluctuate during the $t$-channel evolution. The lowest amplitude is the well-known Odderon consisting of three reggeized gluons. In the present paper we have discussed the four-gluon amplitude. We have computed the coupling of four gluons to the $\gamma \rightarrow \eta_{c}$ impact factor and to a baryonic impact factor. Using these results we have found exact solutions for the corresponding four-gluon amplitudes as superpositions of three-gluon amplitudes. In each term of that superposition a pair of gluons merges and behaves like a single gluon in the three-gluon amplitude. This reggeization happens in exactly the same way as in the amplitudes in the $C$-even sector, strengthening the evidence for the universality of this mechanism. Our result implies that there is no direct coupling of an actual four-gluon state to the relevant impact factors at the leading logarithmic level. Instead, the analytic properties of the $C$-odd four-gluon amplitude in the EGLLA are fully determined by those of the three-gluon amplitude.

It has been found that in the $C$-even channel the EGLLA is dominated by exchanges with even numbers of gluons. More precisely, all amplitudes with odd numbers of gluons in the $t$-channel reggeize and become superpositions of amplitudes with even numbers of gluons. Hence the energy dependence is fully given by the spectrum of the $n$-gluon states with even $n$. Correspondingly, the $C$-even amplitudes can be cast into the form of an effective field theory in which only even $n$-gluon states occur which couple to each other via effective transition vertices $V_{2 \rightarrow 2 l}$ 24]. Moreover, it has been observed that the $n$-gluon states as well as the effective transition vertices are conformally invariant in two-dimensional impact parameter space. The obvious goal is to identify that effective conformal field theory of reggeized gluons. Note that this structure of an effective field theory emerges only if one takes into account subleading terms in $N_{c}$ in the $n$-gluon amplitudes. The leading contributions in the expansion in $1 / N_{c}$ reggeize completely when coupled to a photon-impact factor, see for example 23] and [24]. In order to study the interesting possibility of an effective conformal field theory of high energy QCD it is therefore crucial to go beyond the large- $N_{c}$ approximation.

The picture of an effective field theory of reggeized gluons in the $C$-even channel suggests that a similar structure should also emerge in the $C$-odd channel. Our results indicate that in the $C$-odd channel only states with odd numbers of gluons occur, and that here the amplitudes with even number of gluons like $F_{4}$ are superpositions 
of amplitudes with odd numbers of gluons. In that sense the study of the $C$-odd amplitudes is complementary to the investigation of the $C$-even channel. We expect that in the $C$-odd five-gluon amplitude a new effective three-to-five gluon vertex should appear. In the present paper we have shown the main condition for this to be fulfilled, namely the reggeization of the gluon in the $C$-odd amplitudes. Recall that the transition kernels $K_{2 \rightarrow m}$ in the integral equations of the EGLLA always start from two gluons. We therefore expect that the three-to-five transition proceeds via independent two-to-four gluon transitions in each gluon pair. Since in the three-gluon amplitude each pair of gluons is in a symmetric color octet state that would give us something like an effective two-to-four gluon vertex in the symmetric octet. This in turn would be an element of the effective theory which should play an important role also in the $C$-even channel, for example in the transition from the four- to the six-gluon state. The study of the $C$-odd five-gluon amplitude would therefore contribute to a better understanding of the general picture of the effective field theory of the color glass condensate.

The analysis of the integral equation for the five-gluon amplitude will clearly require the knowledge of the corresponding impact factor. In the present paper we have given an explicit formula for it in the case of the $\gamma \rightarrow \eta_{c}$ impact factor. In that calculation we have found different representations which are equivalent. In the approach to the integral equations developed in [24, however, the impact factor is promoted to an ansatz for the reggeizing part of the full amplitude. In that step, the different representations for the impact factor will in general yield different results, posing a potential problem for the further analysis of the integral equations. The origin of the different representations was the fact that in the $\gamma \rightarrow \eta_{c}$ impact factor the gluons come naturally in two groups according to whether they are coupled to the quark or the antiquark, but on the other hand we need to split them into three groups for having three momentum arguments of the Odderon amplitude. This problem can be avoided by using baryonic impact factors instead. Therefore we consider it an important future project to generalize our analysis of the baryonic impact factor to arbitrary numbers of gluons. This would give us more information on the reggeization of the gluon and might be interesting also in the $C$-even channel.

The knowledge of the three-to-five gluon transition in the effective field theory for the color glass condensate would be very interesting from several points of view. It would for example allow one to compute the splitting of an Odderon into an Odderon plus a Pomeron. From the theoretical perspective, one could compare it with the known splitting vertex of the Pomeron into two Odderons and hence study the properties of the effective vertices under crossing. Also from a phenomenological perspective it would be useful to compute that vertex. Clearly, it would help us in understanding the interplay of Pomeron and Odderon exchanges in high energy reactions. Further, it would also make it possible to compute the effect of Pomeron loops on the Odderon intercept, and to study saturation effects for the Odderon in the large- $N_{c}$ limit.

\section{Acknowledgements}

We would like to thank O. Nachtmann and S. Wallon for helpful discussions. C. E. was supported by the Bundesministerium für Bildung und Forschung, projects HD 05HT1VHA/0 and HD 05HT4VHA/0, and by a Feodor Lynen fellowship of the Alexan- 
der von Humboldt Foundation.

\section{A The Pomeron quark loop with $n$ gluons}

In this appendix we want to consider the $\gamma^{*} \rightarrow \gamma^{*}$ impact factor with $n$ gluons attached. Due to the quantum numbers of the photon the $n$-gluon system is in a $C$-even state and hence contributes to the Pomeron channel. This particular impact factor was already considered in [24] for up to six gluons in the context of the EGLLA. In [19] the same impact factor was studied in the simpler case of the large- $N_{c}$ limit. The considerations in the present paper make it now straightforward to obtain the general form of that impact factor for arbitrary gluon numbers.

There are some differences to the $\gamma \rightarrow \eta_{c}$ impact factor. Firstly, the lowest Pomeron state is built of only two gluons and the color tensor is $\delta^{a_{1} a_{2}}$ for this state. The corresponding two-gluon Pomeron quark loop is called $D_{(2 ; 0)}^{a_{1} a_{2}}$ and the momentum part $D_{(2 ; 0)}$ consists of only two diagrams. (Similar to the Odderon case described above, there are originally four diagrams that can be reduced to two when taking into account the symmetry under quark-antiquark exchange.) Another difference concerns the color tensors. Our analysis of the $\gamma \rightarrow \eta_{c}$ impact factor made use of the fact that there is a $\gamma^{5}$ matrix at the meson vertex and therefore a mass term must be chosen in one quark propagator to give a non-vanishing $\gamma$-trace. In the Pomeron case this is no longer true, as there is a $\gamma^{\mu}$ instead of the $\gamma^{5}$ matrix and all propagators therefore lend their momentum parts in order to give a non-vanishing trace (in the limit that the quark mass is negligible compared to the typical longitudinal quark momenta). This implies that now states with an odd number of attached gluons come with $f$ type tensors, whereas states with an even number of gluons come with $d$ type tensors.

The main difference becomes clear when considering the state with three $t$-channel gluons. For the Odderon we already found two different color tensors when we added one gluon to the minimal number of gluons, that is in the four-gluon quark loop. In Pomeron case, on the contrary, the three-gluon Pomeron quark loop has only one color tensor $f^{a_{1} a_{2} a_{3}}$. In complete analogy to (42) the three-gluon Pomeron quark loop can be constructed by considering the three possible combinations of splitting gluons. An $f$ tensor is again contracted with the color tensor of the amplitude:

$$
\begin{aligned}
D_{(3 ; 0)}^{a_{1} a_{2} a_{3}}\left(\mathbf{k}_{1}, \mathbf{k}_{2}, \mathbf{k}_{3}\right)=\frac{g}{2} & {\left[f_{a_{1} a_{2} b_{1}} \delta^{a_{3} b_{2}} D_{(2 ; 0)}^{b_{1} b_{2}}(12,3)\right.} \\
& \left.+f_{a_{1} a_{3} b_{1}} \delta^{a_{2} b_{2}} D_{(2 ; 0)}^{b_{1} b_{2}}(13,2)+f_{a_{2} a_{3} b_{2}} \delta^{a_{1} b_{1}} D_{(2 ; 0)}^{b_{1} b_{2}}(1,23)\right] .
\end{aligned}
$$

Again, the arguments denote the indices of the momenta that enter as a sum in the two gluon amplitude. As $D_{(2 ; 0)}$ is symmetric under exchange of its momentum arguments, we do not need to care about the order of its arguments. Similarly to the Odderon case, the above expression can be written in terms of the momentum part of the lowest impact factor only:

$$
D_{(3 ; 0)}^{a_{1} a_{2} a_{3}}\left(\mathbf{k}_{1}, \mathbf{k}_{2}, \mathbf{k}_{3}\right)=\frac{g}{2} f_{a_{1} a_{2} a_{3}}\left[D_{(2 ; 0)}(12,3)-D_{(2 ; 0)}(13,2)+D_{(2 ; 0)}(1,23)\right] .
$$

In the case of four gluons the amplitude contains two different color structures. The corresponding momentum structures are again abbreviated as in the Odderon case in 
(411),

$$
\begin{aligned}
D_{(4 ; 0)}^{a_{1} a_{2} a_{3} a_{4}}\left(\mathbf{k}_{1}, \mathbf{k}_{2}, \mathbf{k}_{3}, \mathbf{k}_{4}\right)= & -g^{2} d^{a_{1} a_{2} a_{3} a_{4}}\left[D_{(2 ; 0)}(123,4)+D_{(2 ; 0)}(1,234)-D_{(2 ; 0)}(14,23)\right] \\
& -g^{2} d^{a_{2} a_{1} a_{3} a_{4}}\left[D_{(2 ; 0)}(134,2)+D_{(2 ; 0)}(124,3)-D_{(2 ; 0)}(12,34)\right. \\
& \left.-D_{(2 ; 0)}(13,24)\right] \\
\equiv & d^{a_{1} a_{2} a_{3} a_{4}} D_{(4 ; 0)}^{(1)}+d^{a_{2} a_{1} a_{3} a_{4}} D_{(4 ; 0)}^{(2)}
\end{aligned}
$$

The $n$-gluon quark loop for the Pomeron $(n \geq 4)$ is then constructed in complete analogy to the Odderon quark loop in (55) as a superposition of these terms $D_{(4 ; 0)}^{(1)}$ and $D_{(4 ; 0)}^{(2)}$. Clearly, the tensor - if now appears in the odd and the $d$ tensors in the even gluon-number amplitudes. These results are in complete agreement with the ones found in [24] for up to $n=6$.

\section{References}

[1] L. D. McLerran and R. Venugopalan, Phys. Rev. D 49 (1994) 2233 arXiv:hep-ph/9309289.

[2] L. D. McLerran and R. Venugopalan, Phys. Rev. D 49 (1994) 3352 arXiv:hep-ph/9311205.

[3] E. Iancu and R. Venugopalan, in Quark-Gluon Plasma 3, Eds. R. C. Hwa and X.-N. Wang, World Scientific (2003), arXiv:hep-ph/0303204.

[4] I. Balitsky, Nucl. Phys. B 463 (1996) 99 arXiv:hep-ph/9509348.

[5] A. H. Mueller, Nucl. Phys. B 415 (1994) 373.

[6] A. H. Mueller and B. Patel, Nucl. Phys. B 425 (1994) 471 arXiv:hep-ph/9403256.

[7] I. Balitsky, Phys. Rev. D 60 (1999) 014020 arXiv:hep-ph/9812311.

[8] Y. V. Kovchegov, Phys. Rev. D 60 (1999) 034008 arXiv:hep-ph/9901281.

[9] Y. V. Kovchegov, Phys. Rev. D 61 (2000) 074018 arXiv:hep-ph/9905214.

[10] J. Bartels, Nucl. Phys. B 151 (1979) 293.

[11] J. Bartels, Nucl. Phys. B 175 (1980) 365.

[12] J. Bartels, DESY 91-074 (unpublished)

[13] J. Kwieciński and M. Praszałowicz, Phys. Lett. B 94 (1980) 413.

[14] E. A. Kuraev, L. N. Lipatov and V. S. Fadin, Sov. Phys. JETP 45 (1977) 199 [Zh. Eksp. Teor. Fiz. 72 (1977) 377].

[15] I. I. Balitsky and L. N. Lipatov, Sov. J. Nucl. Phys. 28 (1978) 822 [Yad. Fiz. 28 (1978) 1597].

[16] J. Bartels, Phys. Lett. B 298 (1993) 204. 
[17] J. Bartels, Z. Phys. C 60 (1993) 471.

[18] J. Bartels and M. Wüsthoff, Z. Phys. C 66 (1995) 157.

[19] M. Braun, Z. Phys. C 71 (1996) 601 arXiv:hep-ph/9502403.

[20] J. Bartels, L. N. Lipatov and M. Wüsthoff, Nucl. Phys. B 464 (1996) 298 arXiv:hep-ph/9509303.

[21] H. Lotter, Ph.D. Thesis, Hamburg University 1996, DESY 96-262, arXiv:hep-ph/9705288

[22] M. A. Braun and G. P. Vacca, Eur. Phys. J. C 6 (1999) 147 arXiv:hep-ph/9711486.

[23] M. Braun, Eur. Phys. J. C 6 (1999) 321 arXiv:hep-ph/9706373.

[24] J. Bartels and C. Ewerz, JHEP 9909 (1999) 026 arXiv:hep-ph/9908454.

[25] C. Ewerz, Phys. Lett. B 472 (2000) 135 arXiv:hep-ph/9911225.

[26] C. Ewerz, JHEP 0104 (2001) 031 arXiv:hep-ph/0103260.

[27] C. Ewerz, Phys. Lett. B 512 (2001) 239 arXiv:hep-ph/0105181.

[28] C. Ewerz and V. Schatz, Nucl. Phys. A 736 (2004) 371 arXiv:hep-ph/0308056.

[29] J. Bartels, M. Braun and G. P. Vacca, arXiv:hep-ph/0412218

[30] J. Bartels, L. N. Lipatov and G. P. Vacca, Nucl. Phys. B 706 (2005) 391 arXiv:hep-ph/0404110.

[31] L. N. Lipatov, Sov. Phys. JETP 63 (1986) 904 [Zh. Eksp. Teor. Fiz. 90 (1986) 1536].

[32] L. N. Lipatov, Sov. J. Nucl. Phys. 23 (1976) 338 [Yad. Fiz. 23 (1976) 642].

[33] R. A. Janik and J. Wosiek, Phys. Rev. Lett. $82 \quad$ (1999) 1092 arXiv:hep-th/9802100.

[34] J. Bartels, L. N. Lipatov and G. P. Vacca, Phys. Lett. B 477 (2000) 178 arXiv:hep-ph/9912423.

[35] Y. V. Kovchegov, L. Szymanowski and S. Wallon, Phys. Lett. B 586 (2004) 267 arXiv:hep-ph/0309281.

[36] C. Ewerz, arXiv:hep-ph/0306137

[37] S. J. Brodsky, I. Schmidt and J. J. Yang, Phys. Rev. D 70 (2004) 116003 arXiv:hep-ph/0409279.

[38] J. Czyzewski, J. Kwieciński, L. Motyka and M. Sadzikowski, Phys. Lett. B 398 (1997) 400 [Erratum-ibid. B 411 (1997) 402] arXiv:hep-ph/9611225. 
[39] R. Engel, D. Y. Ivanov, R. Kirschner and L. Szymanowski, Eur. Phys. J. C 4 (1998) 93 arXiv:hep-ph/9707362.

[40] J. Bartels, M. A. Braun, D. Colferai and G. P. Vacca, Eur. Phys. J. C 20 (2001) 323 arXiv:hep-ph/0102221.

[41] L. Motyka and J. Kwieciński, Phys. Rev. D $58 \quad$ (1998) 117501 arXiv:hep-ph/9802278.

[42] S. Braunewell and C. Ewerz, Phys. Rev. D $70 \quad(2004) 014021$ arXiv:hep-ph/0403197.

[43] L. N. Lipatov, arXiv:hep-th/9311037.

[44] L. N. Lipatov, JETP Lett. 59 (1994) 596 [Pisma Zh. Eksp. Teor. Fiz. 59 (1994) 571]

[45] L. D. Faddeev and G. P. Korchemsky, Phys. Lett. B 342 (1995) 311 arXiv:hep-th/9404173.

[46] L. N. Lipatov and G. V. Frolov, Sov. J. Nucl. Phys. 31 (1971) 333 [Yad. Fiz. 31 (1971) 588].

[47] I. F. Ginzburg, S. L. Panfil and V. G. Serbo, Nucl. Phys. B 284 (1987) 685.

[48] M. Fukugita and J. Kwieciński, Phys. Lett. B 83 (1979) 119.

[49] E. M. Levin and M. G. Ryskin, Phys. Rept. 189 (1990) 267.

[50] H. G. Dosch, C. Ewerz and V. Schatz, Eur. Phys. J. C 24 (2002) 561 arXiv:hep-ph/0201294. 